\title{
Active navigation and orientation-free spatial representations
}

\author{
HONG-JIN SUN, GEORGE S. W. CHAN, and JENNIFER L. CAMPOS \\ McMaster University, Hamilton, Ontario, Canada
}

\begin{abstract}
In this study, we examined the orientation dependency of spatial representations following various learning conditions. We assessed the spatial representations of human participants after they had learned a complex spatial layout via map learning, via navigating within a real environment, or via navigating through a virtual simulation of that environment. Performances were compared between conditions involving (1) multiple- versus single-body orientation, (2) active versus passive learning, and (3) high versus low levels of proprioceptive information. Following learning, the participants were required to produce directional judgments to target landmarks. Results showed that the participants developed orientation-specific spatial representations following map learning and passive learning, as indicated by better performance when tested from the initial learning orientation. These results suggest that neither the number of vantage points nor the level of proprioceptive information experienced are determining factors; rather, it is the active aspect of direct navigation that leads to the development of orientation-free representations.
\end{abstract}

Humans can learn and remember information about environmental spatial layouts through direct means (e.g., by navigating through the environment) or indirect means (e.g., by viewing a map or encoding verbal descriptions). Theoretical and empirical work indicates that there may be multiple ways in which to learn spatial information, which results in different spatial representations (Eichenbaum \& Cohen, 2001; Golledge, 1999). To understand the nature of these representations, it is important to identify the functional distinctions between the different ways in which humans represent spatial information. One such distinction involves the degree to which one's spatial representation is orientation specific, as identified by whether or not the spatial memory is dependent on the original orientation in which the spatial layout was learned.

\section{Orientation Specificity as \\ Determined by Mode of Learning}

One method by which humans obtain information about their environment is by reading a map, interpreting the spatial information, and utilizing this information to navigate in the real world. Levine, Jankovic, and Palij (1982)

This work was supported by grants from the Natural Science and Engineering Research Council of Canada, the Canadian Foundation for Innovation, and Ontario Innovation Trust to H-.J. S. The authors thank Bruce Milliken and Pat Bennett for helpful discussions and comments on an early version of the manuscript and G. Rombough, P. Singh, and D. Zelek for their assistance in collecting data. We also thank three anonymous reviewers for helpful comments on earlier versions of the manuscript. Correspondence concerning this article should be addressed to H.-J. Sun, Department of Psychology, McMaster University, 1280 Main Street West, Hamilton, ON, L8S 4K1 Canada (e-mail: sunhong@ mcmaster.ca). required participants to learn the spatial layout of a path by examining a two-dimensional (2-D) map and, subsequently, indicate the direction of a learned target landmark in the real world. They found that the participants were better at indicating the direction of a target landmark when they were in an orientation that was aligned with the orientation in which they learned the 2-D map than from any other orientation. This phenomenon has been demonstrated repeatedly and is commonly referred to as the alignment effect (Peruch \& Lapin, 1993; Rossano \& Warren, 1989; Warren, Rossano, \& Wear, 1990). Other studies, however, have demonstrated that under certain circumstances, the alignment effect is not observed (Evans \& Pezdek, 1980; Thorndyke \& Hayes-Roth, 1982). In these investigations, the performance of participants who were trained with a 2-D map was compared directly with the performance of those who were trained by navigating through an environment. The results demonstrated that those who had learned the environmental layout via a 2-D map demonstrated an alignment effect, whereas those who had navigated through the environment did not. The navigational group performed equally well from an orientation either aligned or misaligned with the initial starting orientation.

A large number of studies have confirmed such a differential performance when a spatial layout is learned via a map, versus when learning occurs via direct navigation (Evans \& Pezdek, 1980; May, Peruch, \& Savoyant, 1995; Presson, DeLange, \& Hazelrigg, 1989; Presson \& Hazelrigg, 1984; Presson \& Montello, 1994; Roskos-Ewoldsen, McNamara, Shelton, \& Carr, 1998; Sholl, 1987; Thorndyke \& Hayes-Roth, 1982). In general, these studies have shown that when participants learn from a map, their responses are more accurate and/or faster when they are tested from an orientation that is aligned with the orientation in which 
they had learned the environment than when they are tested from an orientation that is misaligned with the orientation in which they had learned the environment. This implies that an orientation-specific representation underlies performance. In contrast, after learning through direct navigation, participants appear to form an orientation-free representation, so that their performance is not dependent on the orientation in which the spatial array was initially encoded (Presson \& Hazelrigg, 1984).

\section{Theories That Account for the Formation of Orientation-Free/Specific Representations}

Attempts have been made to explain when or how orientation-free/specific representations are developed. One theory hypothesizes that participants develop an orientation-free representation only when they are provided with a sufficient amount of information (Evans \& Pezdek, 1980; Thorndyke \& Hayes-Roth, 1982). It is believed that training via a 2-D map exposes the participant to only one presentation of the stimulus from a single vantage point. However, if the participant were to actually navigate through the environment, he or she would be exposed to a succession of presentations of the environment from multiple vantage points over time, providing him or her with a larger quantity of information. Therefore, it is suggested that if the participant has learned the environment from multiple vantage points in the learning phase of the experiment, this would then allow him or her to more quickly and accurately perform such tasks as pointing to a remembered location. This theory will be referred to as the multiple vantage points theory.

The second theory, proposed by Presson and Somerville (1985), hypothesized that the differences observed in performance after learning through direct navigation, as compared with learning through a 2-D map, may be due to the fact that participants have directly interacted with the environment in the former. Presson and Somerville classified interactive, real-world navigation tasks as primary spatial activities. In contrast, a map-reading task may require participants to rely on symbolic representations of the features of the real environment (RE), thus allowing them to experience the environment only indirectly. These tasks were classified as falling under the category of secondary spatial activities. This theory will be referred to as the primary learning theory.

\section{A Critical Analysis of These Two Theories}

Each of the two theories above specifies particular factors that may account for the differences in performance observed following map learning, as compared with direct navigation. However, an in-depth analysis and empirical tests of these two theories are still required.

The multiple vantage points theory distinguishes between different categories of spatial representations on the basis of whether participants experience a single vantage point, as compared with multiple vantage points, with the emphasis resting on the quantitative differences in the information received. When the number of vantage points that one experiences is considered, a number of different sources of information must be taken into account: body orientation, head orientation, and eye orientation. Among the different sources of information, it is most likely that body orientation is the most informative. Although the orientation of the head and eyes provides overall spatial information, it may provide less reliable information regarding orientation within an environment. For instance, participants frequently turn their heads and visually scan an environment when moving forward along a straight path. Consequently, the number of body orientations participants assume during learning could affect whether the resulting spatial representation is orientation free or orientation specific.

The primary learning theory emphasizes that it is the $a c$ tive aspect of navigation that leads to the development of orientation-free spatial representations. One way to test this theory is to directly compare spatial performance between conditions in which participants interact with the environment and conditions in which participants passively view the same visual event. If orientation-free representations are found after active learning, but not after passive learning, this would provide critical support for this theory. Moreover, if the active aspect of spatial learning is really the determining factor in the development of an orientationfree representation, it is important to identify the critical factors involved. Two potential factors are (1) the physical motor behavior typically associated with navigation and (2) the degree of active control over one's movement and the resulting information received. Physical motor behavior consists of a sequence of locomotor activities and the associated proprioceptive/efferent and vestibular information. The degree of active control includes such factors as the allocation of attentional resources and the anticipation of the causal relation that exists between motor output and sensory information. We can assess the contributions of each of these two factors by examining conditions in which the presence of natural locomotor behavior is restricted but active control over navigation is retained. This, in turn, would allow us to determine whether an orientation-free representation would still be observed under these circumstances.

\section{Spatial Representations Following \\ Navigation in a Virtual Environment}

Until recently, most of the studies in which the alignment effect has been examined have required participants to initially learn an environment by either studying a map or directly navigating in the real world. To explore why orientation-specific/free representations occur, it is imperative to develop other learning paradigms that will allow for the independent manipulation of the critical aspects of spatial processing mentioned above. Virtual reality (VR) technology is an ideal candidate for such a spatial-learning paradigm (Loomis, Blascovich, \& Beall, 1999; Wilson, 1997).

Virtual environments (VEs) are designed to simulate our interactions with the real world by providing visual, auditory, and haptic information updated in real time. VR 
setups are able to track participants' actions and simultaneously update the appropriate visual and nonvisual input in response to their movements. The most commonly used input devices include keyboards, mouses, and joysticks (Richardson, Montello, \& Hegarty, 1999; Tlauka \& Wilson, 1996). Advanced VR setups are able to incorporate nonvisual information that is normally available in the real world (e.g., leg or body movements during locomotion), allowing users to interact with the VE in a more natural way (Chance, Gaunet, Beall, \& Loomis, 1998; Kearns, Warren, Duchon, \& Tarr, 2002; Klatzky, Loomis, Beall, Chance, \& Golledge, 1998).

When the alignment effect has been examined specifically, the few empirical studies that have been conducted using desktop VR setups have provided mixed results. Richardson et al. (1999) demonstrated a marginal alignment effect for situations in which learning occurred by navigating in a VE and testing occurred by navigating in the real-world equivalent. Another study by Tlauka and Wilson (1996) directly compared the effects of map learning versus navigationallearning in a VE. It was reported that the alignment effect was not observed in the VE navigationallearning condition but was observed in the map-learning condition.

\section{The Rationale for the Present Study}

The main focus of the present study was to examine the critical factors that lead to the typically observed development of orientation-free representations following real and virtual navigation. Our series of experiments involved having participants learn the spatial layout of a floor of a complex building (in RE, in VE, or through a map). Specifically, the participants learned the locations of a set of landmarks in the environment, after which they were positioned in a location along the learning path that was either aligned or contra-aligned with the orientation in which the path had initially been learned. The participants were then asked to make directional judgments by pointing toward the target landmarks learned along the path. Their spatial performance was assessed through measurements of pointing error and reaction time.

For the VE conditions, the participants navigated through a realistic, immersive, interactive environment containing high-quality visual duplicates of real-world environmental details presented through a head mounted display (HMD). Most importantly, the VR setup included the availability of proprioceptive information by having the participants pedal a stationary bike when navigating. It was expected that the results produced with our high-quality multisensory interface would be comparable to the results produced following real-world navigation.

In Experiment 1, we compared spatial representations obtained through map learning, through navigation in an $\mathrm{RE}$, and through navigation in a VE. As was expected, no alignment effect was observed following either VE navigation or RE navigation but was observed following map learning. However, in this experiment, during navigation, the participants navigated down all the hallways in the environ- ment, and as a result, the overall movement trajectory involved many turns. It remained possible that the orientationfree representations observed in both the VE and the RE conditions may have been a result of the multiple body orientations the participants experienced as they navigated along the path during learning.

In Experiment 2, the participants were required to maintain the same body orientation when navigating through a single path in the same building. Again, orientation-free representations were observed. The results of Experiment 2 indicated that multiple body orientations are not necessary to develop an orientation-free representation, in contrast to the predictions made by the multiple vantage points theory. An alternative explanation for why the participants developed orientation-free representations in navigation conditions is that the active aspect of navigating through an environment affects how participants form spatial representations.

To test this theory, in Experiment 3, we compared conditions in which participants actively navigated through an environment with conditionsin which participants passively viewed the equivalent visual scene. Our results demonstrated that orientation-free representations were observed only when the participants actively navigated through the environment.

To understand the critical componentsin the active aspect of the task, in Experiment 4, we tested whether realistic proprioceptive information is critical in forming orientationfree representations. In this experiment, the participants were provided with two levels of proprioceptive information, by having them either pedal a bike (leg movements) or operate a computer mouse (hand/finger movements). An orientation-free representation was observed in both the bicycle and the mouse conditions. This suggests that minimal proprioceptive information is sufficient to produce an orientation-free representation.

\section{EXPERIMENT 1}

Typical studies in which the alignment effect has been examined using real-world navigation paradigms have been conducted in environments that are the size of a small room, with the paths marked on the floor (Palij, Levine, \& Kahan, 1984; Presson et al., 1989; Presson \& Hazelrigg, 1984; Roskos-Ewoldsen et al., 1998; Sholl, 1987; Sholl \& Bartels, 2002). Only a few studies have tested spatial learning in a navigable large-scale environment beyond room size (Richardson et al., 1999; Rossano, West, Robertson, Wayne, \& Chase, 1999; Sholl, 1987; Thorndyke \& Hayes-Roth, 1982). In addition to the issue of the absolute size of the environment, spatial processing can be affected by whether the whole environment can be processed from a single view. Siegel (1981) recognized that there may be inherent differences in the processing of spatial layouts that can be viewed from a single glance and those that must be learned in segments, from multiple views, and integrated over time. It is important to further investigate spatial learning in large-scale environments that can be learned only 
through sequential exploration-for example, exploring a complex building with multiple hallways.

In the present study, we examined the spatial representations of human participants after they had learned the layout of a floor of a complex building $(200 \times 200 \mathrm{ft})$. Performance following learning the environment via RE navigation was compared with learning the environment via $\mathrm{VE}$ navigation and learning via a map. In all three conditions, the participants were subsequently tested in the RE from a position that was either aligned or contra-aligned with the originally learned orientation. On the basis of a previously established consensus, it was expected that an orientationfree representation would be formed following RE navigation and that an orientation-specific representation would be observed following map learning.

Both the multiple vantage points theory and the primary learning theory would predict that, similar to RE navigation, an orientation-free representation would be formed following VE navigation. That is, because the number of viewpoints experienced would be identical and the degree of active navigation would be comparable for each, one would expect that this would result in the development of similar representations.

\section{Method}

\section{Participants}

Forty-two students from McMaster University (18-25 years of age) were randomly assigned to one of the three learning conditions: the RE condition, the VEcondition, and the map condition. The participants had not previously been exposed to the testing environment, and all had normal or corrected-to-normal visual acuity. The participants were compensated with either course credit or money.

\section{Materials}

RE condition. For the RE condition, navigation took place in the basement of the Kenneth Taylor Hall (KTH) building (about $200 \times$ $200 \mathrm{ft}$ ) on the McMaster University campus. Distinct target landmarks from this environment were used for learning (see Figure 1). The participants estimated the direction of the target landmarks by pointing, and such estimates were measured by a circular floor dial about $2 \times 2 \mathrm{ft}$ in size, marked in increments of $1^{\circ}$.

VE condition. For the VE condition, navigation took place in a virtual simulation of the environment used in the RE condition. The VR interface consisted of a stationary mountain bike. The rear tire of the bike was equipped with an infrared sensor that collected information about the rear tire rotation speed. In addition, a potentialmeter was mounted on the handlebars to detect turning movements. Both speed and turning signals were input into the serial port of an SGI Onyx2 with an InfiniteReality2 Engine. The visual environment was rendered by the SGI computer and was presented to the participants via an HMD (V8, Virtual Research; liquid crystal display with a resolution of $640 \times 480$ pixels per eye and a field of view of $60^{\circ}$ diagonal), with each eye receiving the same input. The exact threedimensional (3-D) layout of the basement of the KTH building was simulated using Open Inventor. Most of the salient landmarks were created by texture-mapping digital photographs of the actual landmarks onto virtual landmarks. Other landmarks in the environment (such as lockers, chairs, doors, bulletin boards, etc.) were created using Showcase drawing software.

Map condition. For the map condition, the participants learned the environment by using an outline of the path of the KTH basement displayed on a 15-in. computer monitor. Locations of the target landmarks were labeled on the map and were accompanied by a picture of the corresponding landmark.

\section{Procedure}

All the experimental conditions consisted of two phases: a learning phase and a testing phase (as will be described below). Prior to the experiment, all the participants were instructed on how to use the floor dial to make a directional estimate.

Navigation conditions: RE versus VE. In the learning phase, the participants learned KTH either by being physically led by the experimenter through the RE or by navigating through a simulated version of KTH by pedaling a stationary mountain bike while being verbally directed by the experimenter's commands of "right" and "left." The participants' pedaling speed in the VE was matched to the average walking speed of the participants who learned KTH in the RE. Before the learning phase, the participants who learned the spatial layout of KTH in the VE received practice in another VE (different from the one used in the experiment) to familiarize themselves with navigating in our VE and handling the equipment.

During the learning phase, seven landmarks along the learning path were used (see Figure 1). As each landmark was pointed out and described by the experimenter, the participants were asked to face the landmark and say aloud the name of the landmark, to ensure that there was no ambiguity with respect to the identity of any of the landmarks.

The same set of target landmarks was presented in each of the two paths used, and these target landmarks were learned either from a position of east heading west (Figure 1A) or from a position of west heading east (Figure 1B). Half of the participants in each condition began learning the environment from Landmark 1, which oriented them east prior to learning, and the other half of the participants began at a position between Landmarks 4 and 5, which oriented them west prior to learning.

Prior to the testing phase, the participants were blindfolded and led to two locations along the learning path, which were designated pointing locations. The participants traveled a convoluted path prior to arriving at each testing location, to ensure that they would not be able to predict their location in the environment before they removed the blindfold and were tested. These two locations were chosen to ensure that all the participants were tested from both a location that was aligned (same orientation as the initially learned orientation) and a location that was contra-aligned (orientation opposite to the initially learned orientation). They were then instructed to remove the blindfold and were prompted to point to the seven target landmarks learned. None of the target landmarks was visible from the pointing location. The participants stood on the center of the dial facing $0^{\circ}$ when making their directional estimates. The angle on the dial corresponding to the participants' pointing direction was recorded.

Map condition. The orientations in which the maps were presented were counterbalanced to control for any biases that may have existed for any particular map configuration. Specifically, half of the participants viewed a map of the KTH basement with the east side at the bottom of the map $\left(\operatorname{map}_{\mathrm{e}}\right)$, whereas the other half viewed the same map, but with the west side at the bottom of the map $\left(\operatorname{map}_{\mathrm{w}}\right)$. The orientations of the maps were equivalent to the initial orientation of the navigation conditions, in that map ${ }_{\mathrm{e}}$ would be analogous to traveling from east to west in the navigation condition and map $_{\mathrm{w}}$ would be analogous to traveling from west to east in the navigation condition.

During the learning phase, the participants sat in front of a computer monitor that displayed the map. The participants were required to remember the map and to learn the location of the target landmarks on the map. The experimenter first named each of the target landmarks, and the participants were required to verbally repeat the names back to the experimenter. The order in which the landmarks were learned was the same as that in the corresponding navigation condition. Afterward, the participants were given $3 \mathrm{~min}$ to closely examine the map on their own.

During the testing phase, the participants were blindfolded and taken to the two designated pointing locations in the RE. The participants were then presented with a map similar to the one used in 

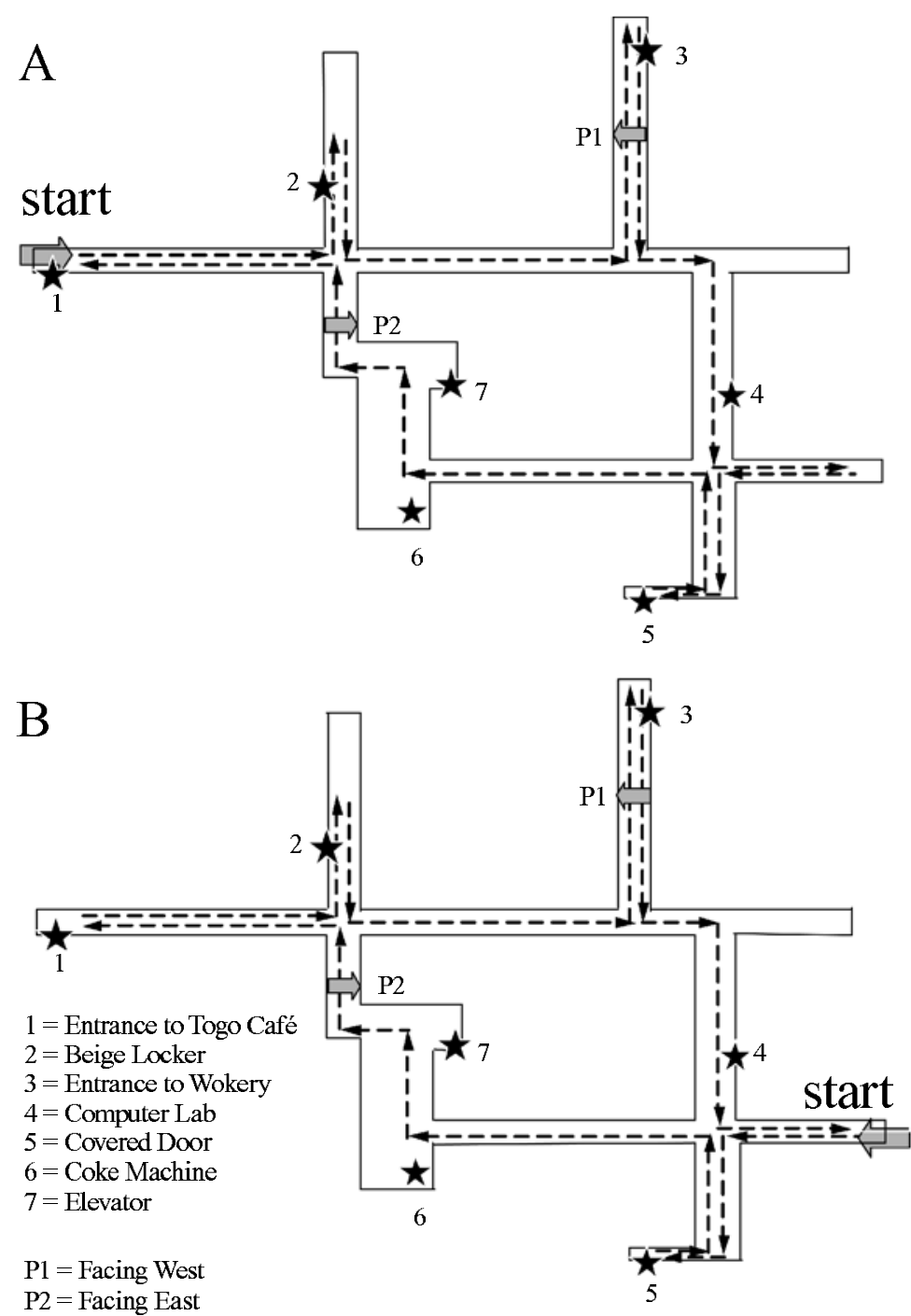

Figure 1. (A) Experiment 1: Learning Path One, starting orientation facing east. The dashed arrow indicates the trajectory and travel direction of the learning path. Each star represents the location of one of the four target landmarks. The two positions and orientations from which the participants were tested are represented by the small block arrows (P1 and P2). (B) Experiment 1: Learning Path Two, starting orientation facing west.

the learning phase in order to orient them within the learned environment. No target landmarks were presented on this map, but a small arrow was drawn as an indicator of the participants' current position and facing direction. The participants were then prompted to point to the seven learned target landmarks in the same way as in the navigation conditions (using the dial).

\section{Results}

For all three conditions (RE, VE, and map), there was no significant difference between starting locations [i.e., whether the participants started from the east heading west or started from the west heading east or whether they learned $\operatorname{map}_{\mathrm{e}}$ or $\left.\operatorname{map}_{\mathrm{w}} ; F(1,36)=0.874, p>.1\right]$, and therefore, the data were collapsed across starting locations.
The raw score distribution of absolute pointing errors for the RE, VE, and map conditions are shown in Figures $2 \mathrm{~A}, 2 \mathrm{~B}$, and $2 \mathrm{C}$, respectively. Absolute pointing errors were defined as the absolute difference between the participants' pointing responses and the correct pointing responses. The distributions of all conditions were unimodal, with the majority of errors falling within the range of $0^{\circ}-$ $30^{\circ}$. There were some differences in performance between aligned and contra-aligned orientations, and such differences were most apparent in the map condition, as compared with the other two conditions.

The distribution of errors observed in the map condition when the participants were tested from a contra-aligned orientation (Figure 2C) was more dispersed, as compared 
A

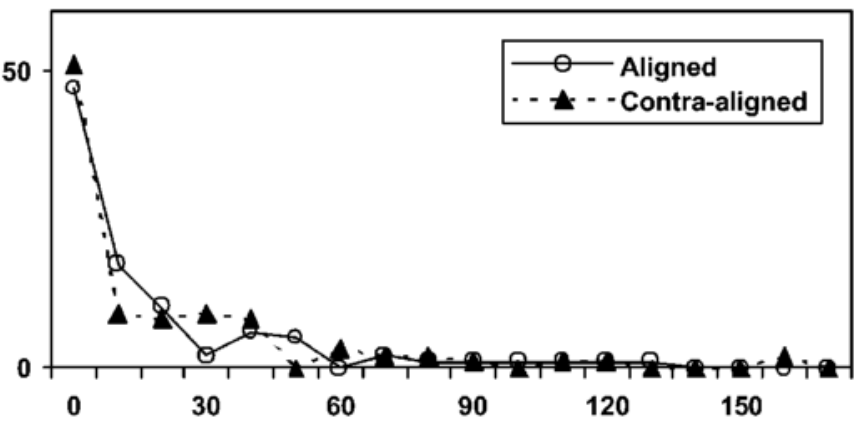

B

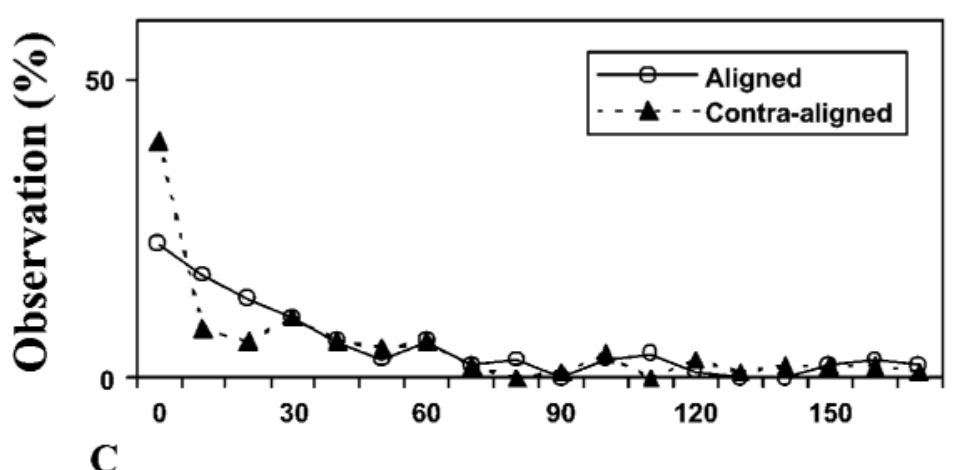

C

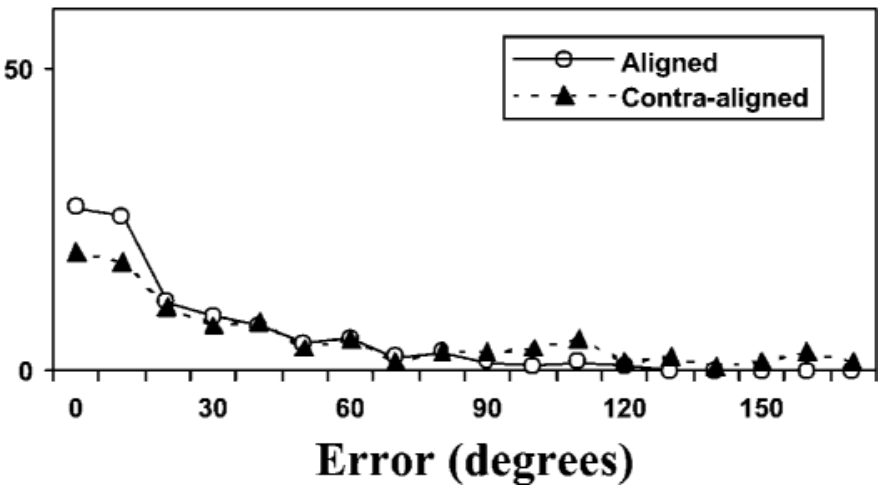

Figure 2. Experiment 1: pointing error distributions for (A) the real environment condition, $(B)$ the virtual environment condition, and $(C)$ the map condition.

with when they were tested from an aligned orientation. The observed distribution is best described as being multimodal, with scattered clusters of large errors. Although some errors were close to $180^{\circ}$, the majority of the errors were distributed variably between $0^{\circ}$ and $180^{\circ}$. There was no apparent peak in response error centering around $180^{\circ}$.

Absolute pointing errors were subject to a log transformation to normalize the distribution before statistical analysis. A $3 \times 2$ between-subjects analysis of variance (ANOVA; three learning conditions [RE learning, VE learning, and map learning] and two testing orientations [aligned and contra-aligned]) was conducted on the mean $\log$ pointing error. Figure 3 illustrates the mean log pointing error for each learning condition and each testing orienta- tion, collapsed across participants in each learning condition and in each testing orientation.

A main effect of learning condition was observed $\left[F(2,44)=8.95, M S_{\mathrm{e}}=0.10, p<.01 ; M_{\mathrm{RE}}=1.04, M_{\mathrm{VE}}=\right.$ $\left.1.32, M_{\mathrm{MAP}}=1.36\right]$. A main effect of orientation was not observed $\left[F(1,44)=0.37, M S_{\mathrm{e}}=0.03, p>.05 ; M_{\text {aligned }}=1.23\right.$, $\left.M_{\text {contra-aligned }}=1.25\right]$. However, an interaction effect between learning condition and orientation was observed $[F(2,44)=$ $\left.7.22, M S_{\mathrm{e}}=0.03, p<.05\right]$. A Tukey post hoc test of comparison was conducted between aligned orientation and contra-aligned orientation for each condition. A significant difference was observed only in the map condition $(p<$ .001 ), but not in either the RE or the VE condition. A Tukey test of comparison was also conducted for learning con- 


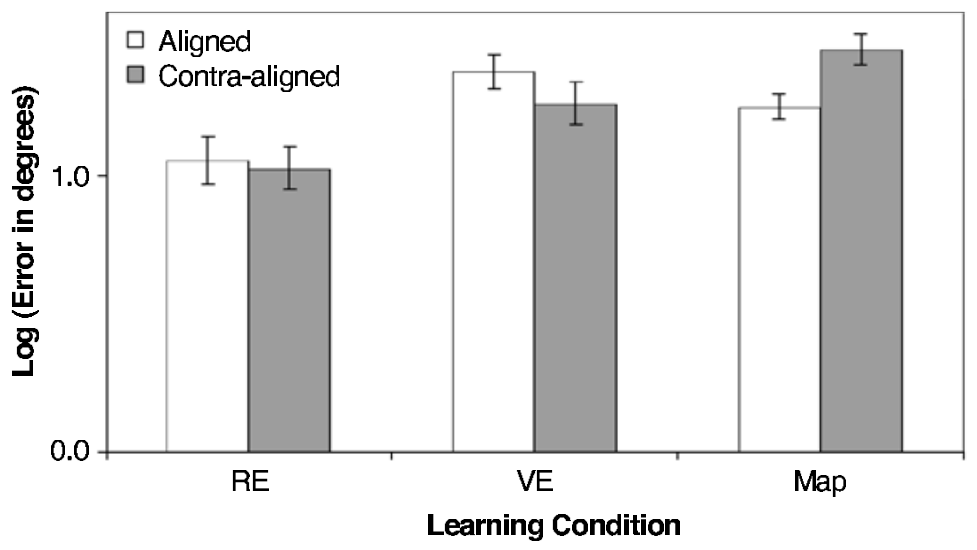

Figure 3. Experiment 1: mean log pointing errors for the real environment (RE), the virtual environment (VE), and the map conditions. Error bars indicate standard errors.

dition, indicating that the errors observed in the RE condition were significantly smaller than the errors observed in the VE or the map condition. There was no significant difference observed between the VE and the map conditions.

\section{Discussion}

The results of Experiment 1 suggest that orientationfree representations were developed following navigational learning in both the RE condition and the VE condition. These findings contrast sharply with the strong alignment effect observed in the map-learning condition. Not only do the orientation-free representations developed during RE navigational learning remain consistent with what is commonly shown in the literature (e.g., Sholl, 1987; Thorndyke \& Hayes-Roth, 1982), but also our results indicate that this same form of orientation-free representation is developed through learning in a VE.

The findings observed for the VE condition differ from past results reported by Richardson et al. (1999), who found a marginal alignment effect $(p<.1)$ for situations in which learning occurred by navigating in a VE and testing occurred by navigating in the real world equivalent. A number of explanations could account for the differences observed in the two studies. In Richardson et al.'s study, the participants were required to estimate route distance and straight-line distance, as well as target direction. In contrast, in our study, the participants were required only to estimate direction. The differences in task requirements could have resulted in the participants' employing different strategies for retrieving spatial information.

A further explanation for the differing results may relate to the qualitative differences that exist between the VR simulations used in the present study and the desktop system used in Richardson et al. (1999). The visual simulation presented in the present experiment included highquality visual graphics, including digital images of real landmarks textured-mapped onto 3-D shapes. Rich environmental textures were also included in order to enable the participants to use optic flow information to continu- ously monitor distance traveled and direction of movement experienced. The visual environment was experienced via an HMD, thus increasing the level of presence the participants experienced. The HMD also allowed for a larger viewing angle than would a typical computer monitor.

In the navigation conditions in Experiment 1, the amount of error observed following learning in the VE was higher, as compared with the error observed following learning in the RE. However, because testing was always conducted in the RE, it is possible that these results reflected a difficulty in transferring the spatial knowledge obtained from a VE to an RE.

With regard to the map condition, when participants were tested from a contra-aligned orientation, it is conceivable that some participants may have had difficulty placing themselves in a position opposite to their initial learning orientation, thus resulting in pointing responses aimed in a direction exactly opposite to that for the target objects (producing errors of around $180^{\circ}$ ). It could be, in fact, that such observations reveal deficits in accessing the correct representation, rather than deficits in developing an accurate representation. The fact that the pointing error distribution did not peak around $180^{\circ}$ (see Figure 2C) indicates that, for the most part, when tested from a contra-aligned orientation, a representation bias is observed, rather than a bias in accessibility.

\section{EXPERIMENT 2}

Evans and Pezdek (1980) have suggested that successive presentations of multiple vantage points during navigation through an RE is important for the formation of an orientation-free representation. One issue that remains to be clarified is whether it is necessary for participants to experience multiple body orientations in order to form orientation-free representations.

In Experiment 1, the learning paths consisted of multiple straight paths connected by $90^{\circ}$ turns. As a result, the participants' heading orientation at any moment was not 
necessarily consistent with their initial orientation. Furthermore, the participants may have encountered particular locations multiple times from different directions. For these reasons, navigation involving multiple turns may result in the encoding of either one orientation-free representation or multiple orientation-specific representations. In other words, it is possible that the design of Experiment 1 masked the presence of an alignment effect by facilitating the encoding of multiple orientation-specific representations as a result of the multiple body orientations the participants assumed during the learning phase. To control for this, it is important to examine whether navigation through paths that do not involve turning the body still leads to orientationfree representations.

Presson, DeLange, and Hazelrigg (1987) examined this problem by testing the effect of being presented with a spatial layout from a single orientation during blindfolded walking in a small environment (less than $10 \times 10 \mathrm{ft}$ ). They found that after participants had learned an environment while maintaining a single body and head orientation, orientation-specific representations were observed. In contrast, the participants formed orientation-free representations after experiencing multiple orientations while learning the path. Therefore, experiencing multiple orientations by turning while walking appeared to be necessary for blindfolded participants to form orientation-free representations during navigation.

In Experiment 2, we tested whether an orientation-free representation would still be observed should participants maintain a uniform body orientation along the entire path during sighted navigation. To examine this issue, we implemented a navigation task that required the participants to travel in a single heading direction. Such a learning paradigm was expected to be much more sensitive to an alignment effect. To avoid the increased demand required to transfer knowledge between learning and testing modes, as may have been the case in Experiment 1, in Experiment 2 both training and testing were conducted in the same mode. Consequently, each of the participants both learned and was tested in the VE and also learned and was tested in the RE.

\section{Method}

\section{Materials}

The equipment used was the same as that used in the VE and RE conditions of Experiment 1. The paths used in this experiment made up a portion of the entire environment used in Experiment 1.

\section{Participants}

Twenty-four undergraduate students from McMaster University participated in this study and were compensated with either course credit or money. All had normal or corrected-to-normal visual acuity.

\section{Procedure}

All the participants completed two conditions: (1) learning and testing in the RE and (2) learning and testing in the VE. Four possible paths in KTH were used: Two horizontal (east-west) paths (H1 and H2) and two vertical (north-south) paths (V1 and V2; see Figure 4). These four paths were included as a portion of the learning path used in Experiment 1 . The lengths of the paths were roughly matched, so that each of the horizontal paths covered a distance of $156 \mathrm{ft}$ and each of the vertical paths covered a distance of $135 \mathrm{ft}$. The horizontal paths were perfectly straight, whereas the two vertical paths included a small, brief, horizontal deviation, which resulted in a $90^{\circ}$ left turn followed by a $90^{\circ}$ right turn. All the participants learned and were tested in the RE, using one of the horizontal paths (e.g., H1) and one of the vertical paths (e.g., V1). All the participants also learned and were tested in the VE, using the remaining horizontal (e.g., H2) and vertical (e.g., V2) paths. For half of the participants, $\mathrm{V} 1 / \mathrm{H} 1$ were tested in the RE, whereas $\mathrm{V} 2 / \mathrm{H} 2$ were tested in the $\mathrm{VE}$, and vice versa for the remaining participants. Furthermore, the order of the environments, the order of the testing locations (path chosen), and the order of the target landmarks were pseudorandomized.

Prior to learning, the participants were given practice and feedback on the pointing task in both the RE and the VE conditions and were trained to effectively navigate in the VE. A single starting position was used, and an invariable direction of travel for each path was maintained. For $\mathrm{H} 1$ the starting position was at the west end, for $\mathrm{H} 2$ it was at the east end, for V1 it was at the south end, and for V2 it was at the north end. For each learning path, the testing landmarks were located on paths that intersected the training path perpendicularly (see Figure 4 for landmark locations and identities).

Each learning trial required the participants to travel along a particular path once from beginning to end. The participants were required to maintain a constant body orientation (facing movement direction) throughout the travel, although they had to turn their heads to view various landmarks in the environment. As the participants were led along the path, the experimenter stopped at specific locations along the path to point out the target landmarks. In addition to the target landmarks, there were also distinct prompting landmarks (pictures posted by the experimenter prior to the experiment) placed at the corners of each of the intersections and at the end of each learning path. These prompting landmarks were brought to the attention of the participants by the experimenter during the learning phase. The purpose of providing these prompting landmarks was to assist the participants in recognizing their location along a path once they were brought back to that location some time later during testing.

The testing phase occurred immediately after the completion of the learning phase. There were two testing positions that the participants were brought to on each path: an aligned position and a contraaligned position (Figure 4).

In the RE, between each testing trial, the participants were led blindfolded along a path outside of the testing area that consisted of many disorienting turns, before being brought back to each testing location (disorientation phase). This was to ensure that the participants were unable to update either their location along a path (via nonvisual cues) or their orientation in the environment before taking off the blindfold and beginning the test trial (Sholl \& Bartels, 2002; Sholl \& Nolin, 1997). In the VE condition, the participants were "teleported" to the pointing locations. In order to mimic the amount of proprioceptive information experienced during the disorientation phase in the RE condition, in the VE condition, the participants pedaled backward with no visual input for an equivalent duration. Once the participants reached the pointing locations and regained visual input, they were prompted to familiarize themselves with their position in the environment. The participants were then prompted by the experimenter to point to each of the target landmarks. The method used to measure the participants' pointing estimations in the $\mathrm{RE}$ was the same as that used in Experiment 1 (floor dial). In the VE condition, the participants pointed to the landmarks by turning the bike's handlebars in the desired direction until they believed the landmark's location to be centered in their visual field. In the testing phase for the VE condition, the participants were provided with a crosshair in the middle of the display, to assist in centering/locating their desired response. In addition to pointing direction, the participants' reaction time was also recorded. 


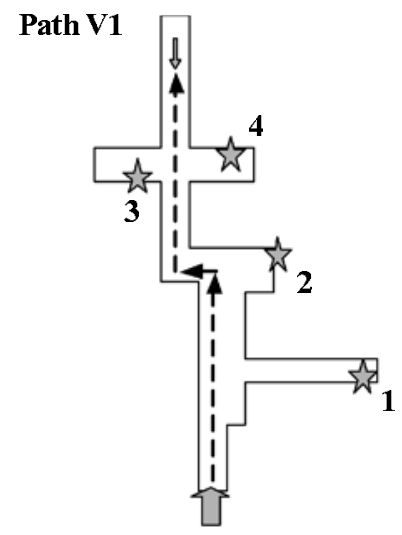

\section{Path H1}

Path H2

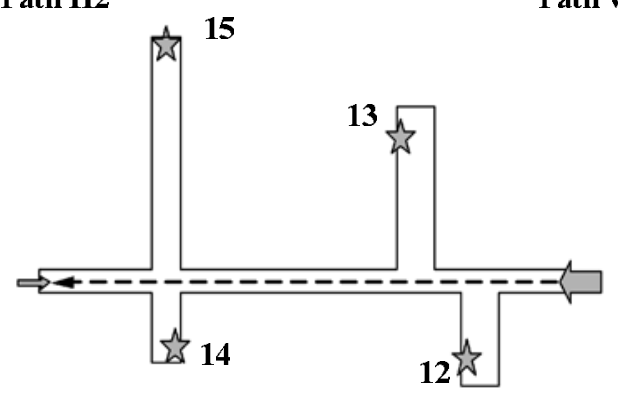

Path V2

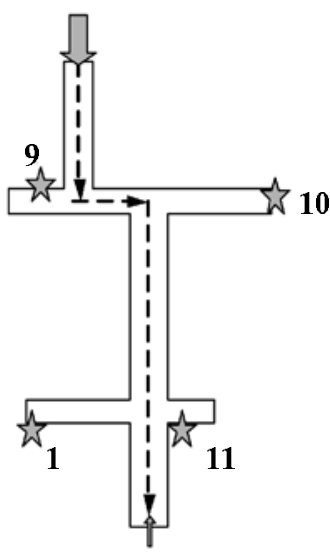

$$
\begin{aligned}
& 8=\text { Water Fountain } 1 \\
& 9=\text { Fire Extinguisher } 2 \\
& 10=\text { Printing Service Sign } \\
& 11=\text { Utility Box } \\
& 12=\text { Blue Billboard } 2 \\
& 13=\text { Water Fountain } 2 \\
& 14=\text { Glass Display Case } 2 \\
& 15=\text { Fish Sign }
\end{aligned}
$$

Figure 4. Experiment 2: The four vertical (V1 and V2) and horizontal (H1 and H2) learning paths, marked with all the corresponding target landmarks (indicated by stars). The orientations from which the participants were tested are indicated by block arrows, with the aligned orientation represented by wider arrows.

\section{Results}

There was no significant difference between the performance of the participants who completed the RE condition first, as compared with those who completed the VE conditionfirst [order effect: $F(1,22)=0.001, p>.1$ ] , and therefore, the data were collapsed across learning order to simplify the results.

The raw score distribution of absolute pointing errors for the RE and VE conditions are shown in Figures 5A and $5 \mathrm{~B}$, respectively. Similarly, the raw score distribution of reaction times for the RE and VE conditions are shown in Figures 5C and 5D, respectively. All the distributions were unimodal. With regard to both errors and reaction times, in both the RE and the VE conditions, the distributionsfor aligned and contra-aligned orientations appear to be quite similar. This served to verify that there is minimal difference across the entire range of errors and reaction times across all the participants.
Both pointing errors and reaction times were subject to a $\log$ transformation to normalize the distribution before statistical analysis. A $2 \times 2$ repeated measures ANOVA $(2$ learning conditions [RE and VE] $\times 2$ testing orientations [aligned and contra-aligned]) was conducted on the mean $\log$ pointing error and the mean log reaction time. Figures 6A and 6B illustrate mean log pointing error and mean log reaction time collapsed across participants in each learning condition and in each testing orientation.

\section{Pointing Errors}

With regard to pointing error, a main effect of learning condition (VE vs. RE) was not observed $[F(1,23)=2.96$, $\left.M S_{\mathrm{e}}=0.07, p=.10 ; M_{\mathrm{RE}}=1.16, M_{\mathrm{VE}}=1.07\right]$. A main effect of orientation was not observed $[F(1,23)=0.44$, $\left.M S_{\mathrm{e}}=0.05, p>.05 ; M_{\text {aligned }}=1.10, M_{\text {contra-aligned }}=1.13\right]$. In addition, an interaction effect between the learning condition and orientation was not observed $[F(1,23)=1.94$, 


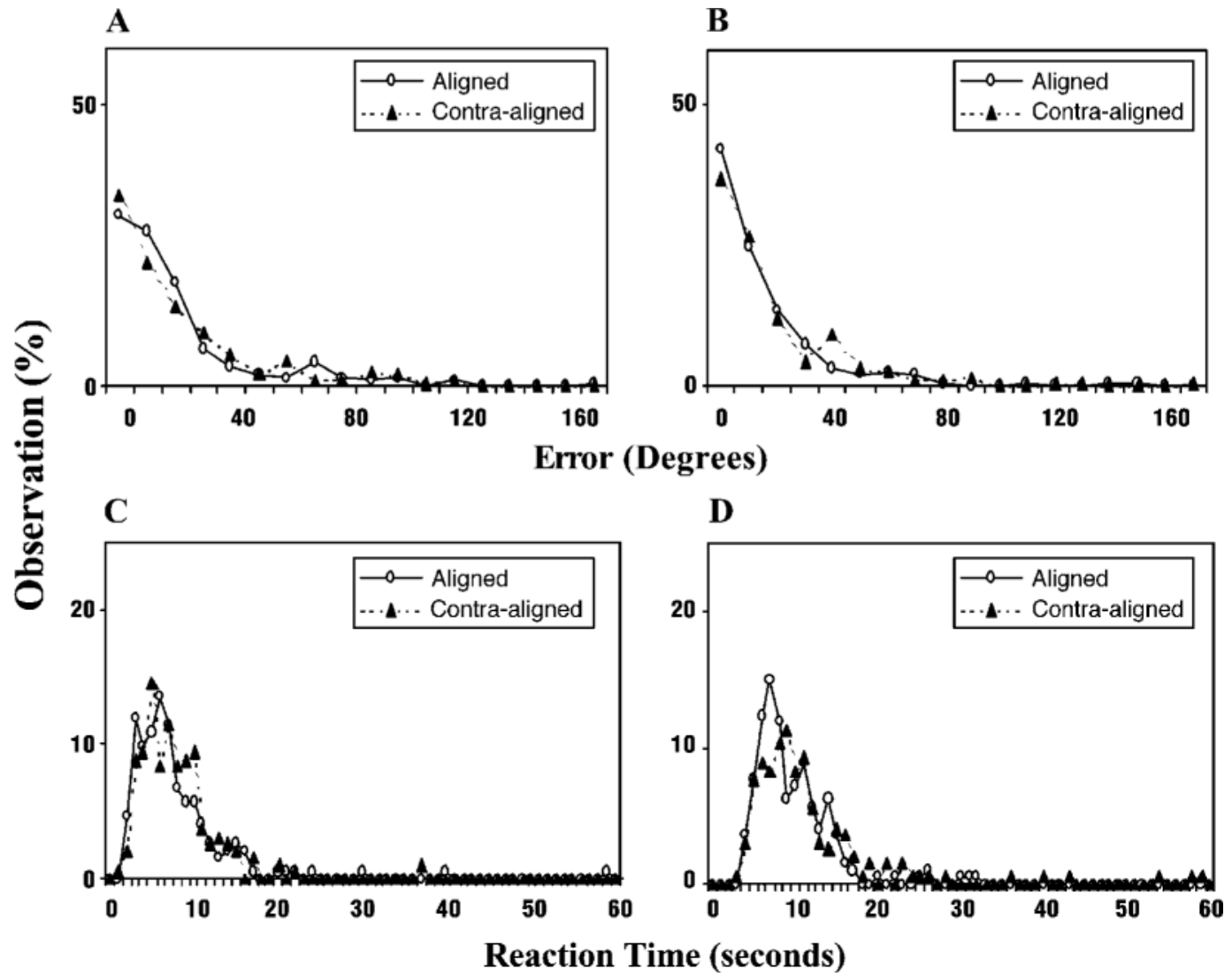

Figure 5. Experiment 2: pointing error distributions for $(A)$ the real environment (RE) condition and (B) the virtual environment (VE) condition and reaction time distributions for $(C)$ the $R E$ condition and (D) the VE condition.

$\left.M S_{\mathrm{e}}=0.03, p>.05\right]$. Therefore, no alignment effect was observed in either the RE or the VE condition.

\section{Reaction Time}

With regard to reaction time, a main effect of learning condition was observed $\left[F(1,23)=21.11, M S_{\mathrm{e}}=0.02, p<\right.$ $\left..01 ; M_{\mathrm{RE}}=0.82, M_{\mathrm{VE}}=0.94\right]$. The participants required significantly longer time to respond in the VE condition than in the RE condition. A main effect of orientation was not observed $\left[F(1,23)=3.55, M S_{\mathrm{e}}=0.01, p=.07 ; M_{\text {aligned }}=0.86\right.$, $\left.M_{\text {contra-aligned }}=0.90\right]$. In addition, an interaction between learning condition and orientation was not observed $[F(1,23)=$ $\left.0.69, M S_{\mathrm{e}}=0.01, p>.05\right]$. Therefore, no alignment effect was observed in either the RE or the VE condition.

\section{Discussion}

The results of Experiment 2 suggest that an orientationfree representation is developed through learning in both an RE and a VE. Orientation-free representations developed through RE navigational learning are consistent with commonly reported results for spatial learning in largescale spaces, even though other studies did not limit the participants' body orientation to one (e.g., Rossano \& Moak,
1998; Rossano et al., 1999; Sholl, 1987; Thorndyke \& Hayes-Roth, 1982).

In Experiment 1 and in many other previous studies in which real-world navigation was used, during the learning phase, participants had the opportunity to assume many different body orientations. Thus, it is reasonable to suspect that they were more likely to build a spatial representation that consisted of multiple orientations. In Experiment 2, inasmuch as the learning path contained primarily a single body orientation, the results suggest that even without being exposed to multiple body orientations during the exploration of the environment, orientation-free representations can be generated. Consequently, Experiment 2 provided a more robust test of the qualities that lead to an orientation-free representation in real-world navigation.

The observed orientation-free representation that resulted following real-world navigation while maintaining a single body orientation is in contrast to the results reported by Presson et al. (1987). They found that when both the body and the head were maintained at a constant orientation, an orientation-specific representation was observed, but if the head and the body did not maintain a constant orientation, an orientation-free representation was observed. 

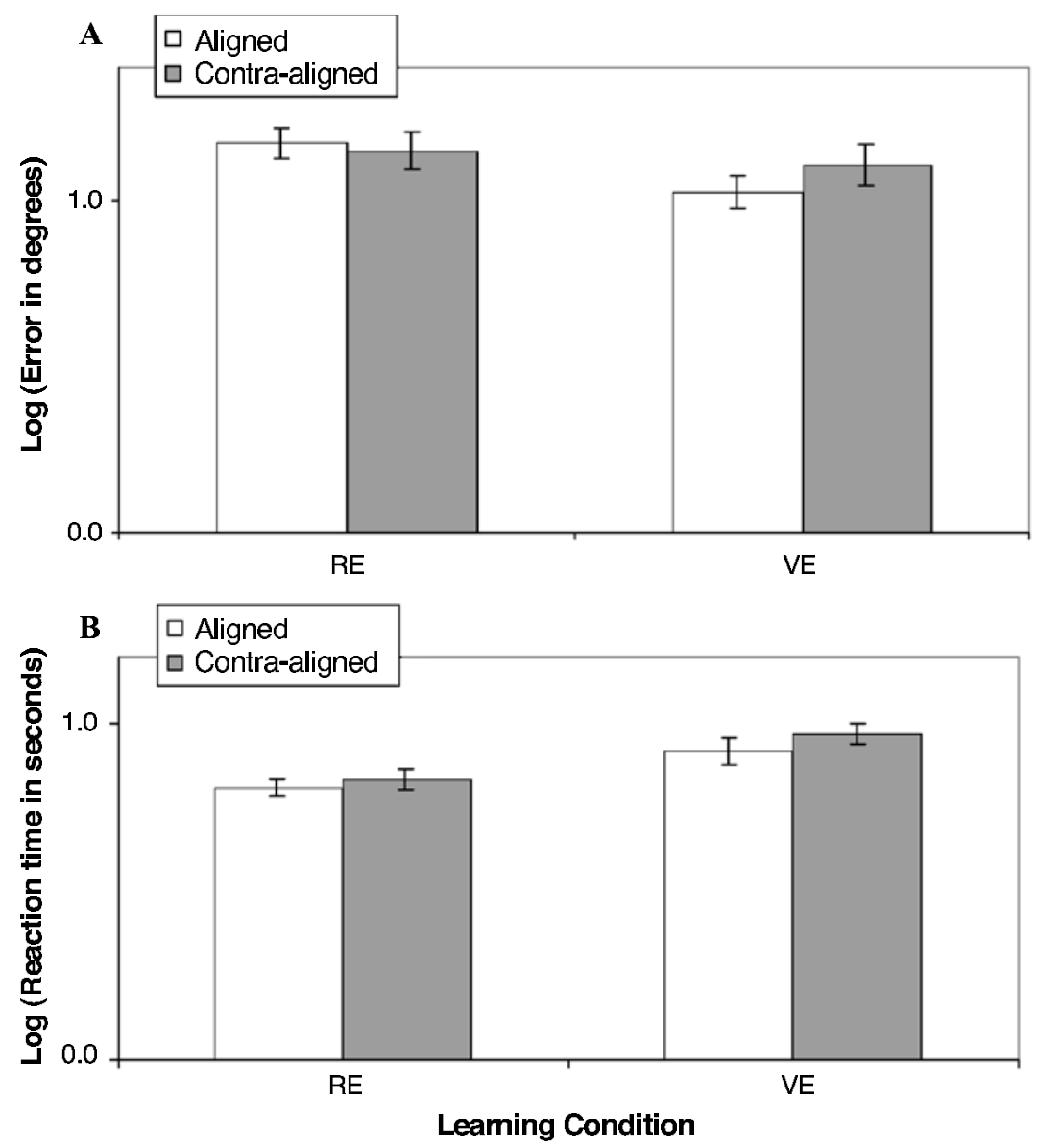

Figure 6. Experiment 2: (A) mean log pointing errors for the real environment (RE) and virtual environment (VE) conditions and $(B)$ mean log reaction times for the $R E$ and VE conditions. Error bars indicate standard errors.

Thus, experiencing multiple orientations by turning while walking seemed to be necessary for blindfolded participants to form orientation-free representations during navigation.

There are a number of differences between their study and ours. First, in their study, the participants had to maintain both a single body and a single head orientation, whereas in our RE condition in Experiment 2, the participants were still able to rotate their head to view landmarks outside their central field of view. In Presson et al.'s (1987) study, the participants were required to walk directly to the location of the landmarks, whereas in our study, the participants viewed the landmarks from a distance. In Presson et al.'s (1987) study, the consequence of limiting body and head orientation was that the participants, at times, were required to walk sideways in order to reach the location of the landmarks, thus forcing the participants to engage in unnatural movements that could potentially affect the way in which spatial layouts were perceived visually and nonvisually. In our experiment, the participants remained on a single path, and their body orientation was in line with the travel direction. Although our design avoided the need for unnatural movement, this resulted in the participants' having to turn their heads. Thus, it remains possible that in our RE condition, it might have been the experience of multiplehead orientations that led to an orientation-free representation.

To further assess the effect of head orientation on spatial performance, we can turn to the results of our VE condition. In some respect, our VE condition shares some common features with Presson's single body/head orientation condition. In our VE condition, the participants changed their view by turning the handlebars of the bike, instead of physically turning their heads, and yet our results showed an orientation-free representation. Consequently, this suggests that multiple head orientations may not be necessary to develop an orientation-free representation.

It is important to note that whereas Presson et al.'s (1987) study required participants to learn the spatial layout through nonvisual sources only, our study provided participants with both visual and nonvisual information. It remains possible that visual learning and kinesthetic learning result in spatial representations that differ.

Despite the difference between our results and Presson et al.'s (1987) results, orientation-free representations were still observed even when the participants were required to 
navigate while maintaining a single body orientation. Consequently, the number of vantage points experienced during navigation may not be the determining factor in whether an orientation-free or an orientation-specific representation will be observed. Therefore, it would be more informative to examine the specific properties of active navigation that may result in different types of spatial representations.

\section{EXPERIMENT 3}

Inasmuch as an alignment effect was not observed in Experiments 1 and 2 in both the RE and the VE conditions, it is important to examine what particular aspects of navigation lead to orientation-free representations. One such factor, as proposed by Presson and Hazelrigg (1984), involves the idea that navigation requires participants to directly interact with the environment. In order to test this theory, one would have to examine situations in which participants do not interact directly with their environments but still passively receive the equivalent spatial information.

Few studies have examined orientation specificity following passive learning. Rossano and Moak (1998) and Rossano et al. (1999) used a computer display to present visual information experienced during navigation. What is interesting is that the resulting spatial representation differed, depending on the way the test environment was presented. If, during test, visual information specifying location was made available, an orientation-free representation was found. However, if the participants were required to simply imagine themselves facing a particular orientation, without any visual or nonvisual feedback, an orientationspecific representation was found.

In Experiment 3, we manipulated the extent to which a participant interacted with the environment and evaluated how this, in turn, affected the type of representation that was observed following learning. We compared conditions in which the participants actively navigated through a VE with conditions in which the participants passively viewed the same visual scene.

\section{Method}

\section{Participants}

Sixteen undergraduate students from McMaster University participated in this study and were compensated with course credit or money. All had normal or corrected-to-normal visual acuity.

\section{Material}

Equipment and paths were the same as those in the VE condition of Experiment 2.

\section{Procedure}

All the participants completed two conditions: (1) the active condition and (2) the passive condition. For both conditions, the learning phase and the testing phase were conducted in the VE. The active condition was essentially the same as the VE condition in Experiment 2. In the passive condition, the participants sat on the bike and wore the HMD, just as in the active condition. They were presented with the same visual scene, recorded during a typical active navigation; however, they did not pedal the bike but, instead, passively viewed the visual display. The participants were provided with the same instructions during learning in both the active and the passive conditions.

All the participants learned and were tested in the active condition, using one of the horizontal paths (e.g., H1) and one of the vertical paths (e.g., V1). All the participants learned and were tested in the passive condition for the remaining horizontal (e.g., H2) and vertical (e.g., V2) paths. For half of the participants, V1/H1 were tested in the active condition, whereas $\mathrm{V} 2 / \mathrm{H} 2$ were tested in the passive condition, and vice versa for the remaining participants. The method used to measure the participants' pointing response estimations was the same as that used in the VE condition of Experiment 2. Pointing direction and reaction time were recorded.

\section{Results}

There was no significant difference between the performance of the participants who completed the active condition first and that of those who completed the passive condition first [order effect: $F(1,14)=0.035, p>.1$ ] , and therefore, the data were collapsed across learning order to simplify the results.

The raw score distribution of absolute pointing errors for the active and passive conditions are shown in Figures 7A and 7B, respectively. Similarly, the raw score distributions of reaction times for the active and passive conditions are shown in Figures 7C and 7D, respectively. All the distributions were unimodal. In the active condition, with regard to both error and reaction time, the distributions for the aligned and the contra-aligned orientations were similar. This served to verify that there was minimal difference across the entire range of pointing errors and reaction times across all the participants. However, in the passive condition, with regard to both errors and reaction times, the distribution for the contra-aligned orientation(Figures 7B and 7D) showed a higher proportion of large errors and a higher proportion of long reaction times, as compared with those for the aligned orientation. There was no apparent peak in response error around $180^{\circ}$, indicating that the participants were not simply unable to adjust for contraaligned judgments.

Both pointing errors and reaction times were subject to a log transformation to normalize the distribution before statistical analysis. A $2 \times 2$ repeated measures ANOVA $(2$ learning conditions [active and passive] $\times 2$ orientations [aligned and contra-aligned]) was conducted on the mean $\log$ pointing error and the mean log reaction time. Figures $8 \mathrm{~A}$ and $8 \mathrm{~B}$ illustrate mean log pointing error and mean $\log$ reaction time collapsed across participants in each learning condition and each testing orientation.

\section{Pointing Error}

With regard to pointing error, a main effect of learning condition was observed $\left[F(1,15)=11.10, M S_{\mathrm{e}}=0.04, p<\right.$ $\left..05 ; M_{\text {Active }}=0.93, M_{\text {Passive }}=1.09\right]$. A main effect of orientation was not observed $\left[F(1,15)=2.97, M S_{\mathrm{e}}=0.03\right.$, $\left.p=.11 ; M_{\text {aligned }}=0.98, M_{\text {contra-aligned }}=1.05\right]$. An interaction between learning condition and orientation was observed $\left[F(1,15)=7.07, M S_{\mathrm{e}}=0.02, p<.05\right]$.

In the active condition, a Tukey post hoc test of comparison between aligned orientation and contra-aligned 
A

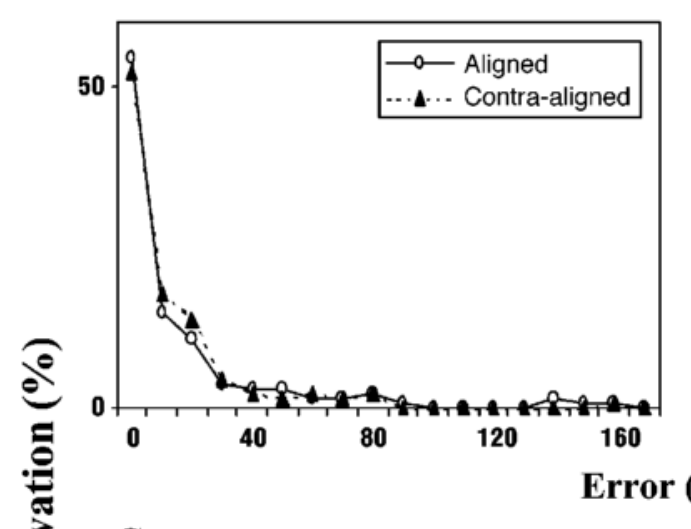

C

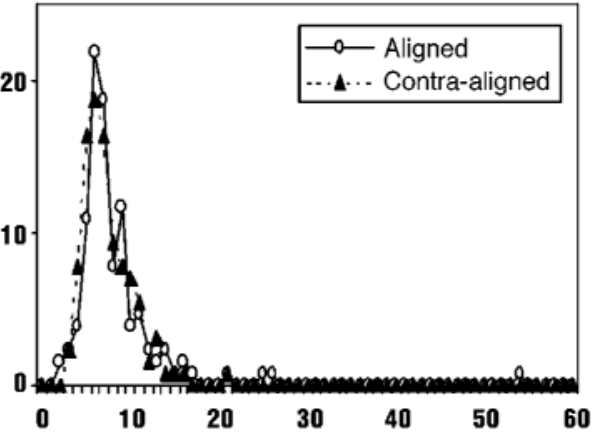

B

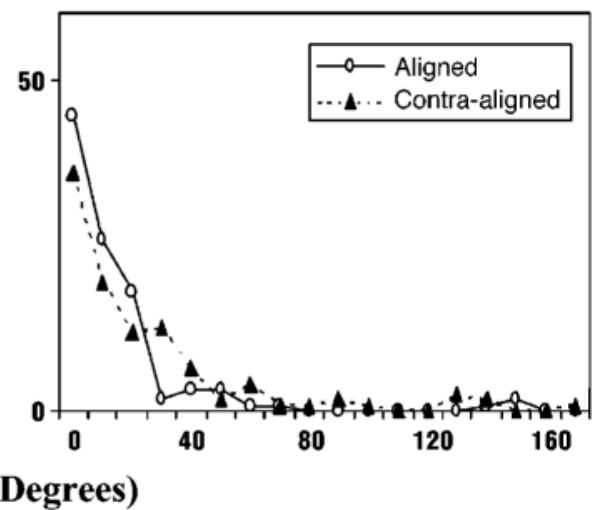

D

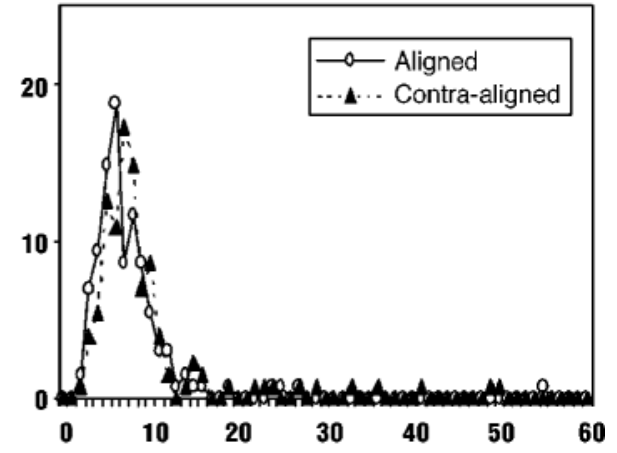

Reaction Time (seconds)

Figure 7. Experiment 3: pointing error distributions for (A) the active condition and (B) the passive condition and reaction time distributions for $(C)$ the active condition and (D) the passive condition.

orientation was not significant, thus failing to demonstrate an alignment effect. However, in the passive condition, a Tukey comparison between aligned orientation and contraaligned orientation was significant. This demonstrated an alignment effect in the passive condition only. Furthermore, a Tukey comparison between the active-aligned orientation and the passive-aligned orientation was not significant.

\section{Reaction Time}

With regard to reaction time, no significant main effect of learning condition was observed $\left[F(1,15)=0.049, M S_{\mathrm{e}}=\right.$ $0.01, p>.05 ; M_{\text {Active }}=0.82, M_{\text {Passive }}=0.83$ ], nor was a main effect of orientation observed $\left[F(1,15)=1.55, M S_{\mathrm{e}}=\right.$ $\left.0.01, p>.05 ; M_{\text {aligned }}=0.81, M_{\text {contra-aligned }}=0.84\right]$. However, a significant interaction effect was observed between learning condition and orientation $\left[F(1,15)=6.45, M S_{\mathrm{e}}=\right.$ $0.01, p<.05]$.

In the active condition, a Tukey post hoc test of comparison between aligned orientation and contra-aligned orientation was not significant, thus failing to demonstrate an alignment effect. In the passive condition, a Tukey comparison between aligned orientation and contra-aligned orientation was significant. This indicated an alignmenteffect in the passive condition only.

\section{Discussion}

Experiment 3 demonstrated that active navigationallearning leads to an orientation-free representation, whereas experiencing the path passively leads to an orientation-specific representation. Across conditions, pointing error and reaction time were highest for the passive condition, when tested from a contra-aligned orientation. However, pointing error and reaction time for both the active and the passive conditions were comparable for an aligned orientation. Therefore, the comparable performance between the passive-aligned and the active-aligned conditions suggests that the observed alignment effect in the passive condition was not due to poor overall performance. This lessens the possibility that during the passive condition, the participants attended less to the spatial information or were less motivated to perform the task.

When people actively navigate, they encode both the visual information of the spatial layout and the nonvisual information that is simultaneously available. The availability of concordant information from multiple inputs may enable participants to develop stronger and more accessible spatial knowledge. Such multisensory interactions may allow one to more effectively integrate spatial information obtained from different views, thus forming a unified 

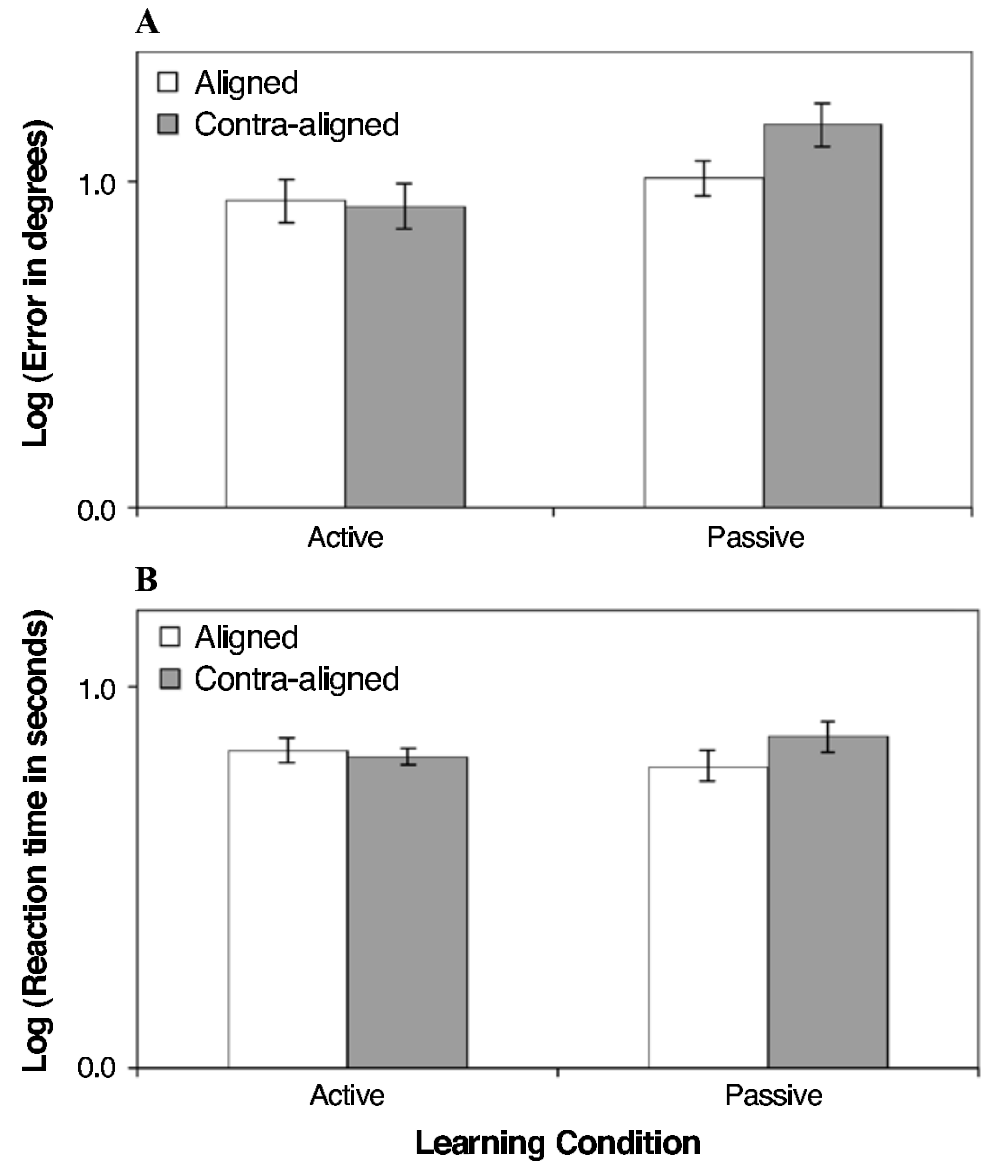

Figure 8. Experiment 3: (A) mean log pointing errors for the active and passive conditions and (B) mean log reaction time for the active and passive conditions. Error bars indicate standard errors.

knowledge of space independently of their own position in space (Presson et al., 1989). In contrast, in the passive condition, when one receives only a visual display of the movement trajectory in the absence of proprioceptive cues, the spatial information gained may be encoded as discrete and specific visual events and may be restricted or biased toward the originally learned orientation (Rossano et al., 1999).

As was previously mentioned, Rossano and Moak (1998) conducted an experiment involving a condition similar to our passive condition. The orientation-free representation observed following passive learning in Rossano and Moak's experiment was in contrast to the results of the present study. It is possible that, in our study, since our participants were traveling only one straight pathway, they were more susceptible to forming a spatial representation related to the orientation of the movement path.

In terms of general spatial performance (not in the context of orientation specificity), few studies have compared performance in a directional pointing task following active learning with performance following passive learning (Wilson, 1999; Wilson, Foreman, Gillett, \& Stanton, 1997). In these studies, investigators failed to observe a difference between active conditions and passive conditions. This is inconsistent with our results, which showed that pointing errors in the active condition were significantly lower than those in the passive conditions. The discrepancy between these results could be due to the type of VR interface and the quality of the visual simulation implemented. For example, whereas the participants from the previous studies used keyboards to navigate in the virtual environment, the participants in our study controlled their navigation in a more natural way (pedaling a bike).

Although overall aligned performance was comparable for both the active condition and the passive condition in the present study, an important difference was revealed when we examined learning in the context of orientation specificitythat is, when the participants were tested from a contraaligned orientation. The alignment effect observed in the passive condition indicates that it is the active nature of navigation that leads to the development of an orientationfree representation. It is therefore important to further understand the mechanisms that underlie active navigation. 


\section{EXPERIMENT 4}

The concept referred to here as active navigation may actually encompass a subset of factors that may potentially influence the spatial representations that one ultimately develops. Intuitively, it would seem that the sensorimotor information available during active navigation would be the determining factor. In a typical navigation task, participants receive proprioceptive information during locomotion.Extensive literature has demonstrated that proprioceptive information is useful for updating egocentric representations of distance traveled, direction of movement, and relative location of nearby landmarks in the real world (Farrell \& Thomson, 1998; Klatzky et al., 1998; Loomis, Klatzky, Golledge, \& Philbeck, 1999; Simons \& Wang, 1998; Sun, Campos, Chan, Young, \& Ellard, 2004) and in VR (Bakker, Werkhoven, \& Passenier, 1999, 2001; Chance et al., 1998; Kearns et al., 2002; Klatzky et al., 1998; Sun, Campos, \& Chan, 2004). A number of studies have examined the question of nonvisual updating in the context of orientation specificity (Easton \& Sholl, 1995; Farrell \& Robertson, 1998; Presson \& Montello, 1994; Rieser, 1989). For example, Rieser required blindfolded participants to point toward remembered target locations. Results demonstrated that directionaljudgments were better when the participants physically moved to the testing location than when they merely imagined themselves positioned in that same testing location. Rieser claimed that when visual information is not available, spatial updating is achieved through the proprioceptive information generated by self-movement. Although proprioceptive information plays a large role when visual information is not available, it remains to be tested whether such information is still important if vision is made available.

The results of Experiment 3 indicated that the complete lack of proprioceptive information led to an orientationspecific representation. It would, therefore, be interesting to test whether different levels of proprioceptive information would lead to different types of spatial representations. For instance, one could compare conditions in which participants are provided with natural proprioceptive information during navigation (e.g., leg movements) with conditions in which only minimal proprioceptive information is provided (e.g., hand movements). Such results would complement findings from the literature on nonvisual updating by identifying the minimal information required to achieve the same nonvisual updating as that revealed in real movement.

In Experiment 4, in order to determine which components of active navigation lead to an orientation-free representation, we compared conditions in which realistic proprioceptive information was available with conditions in which minimal proprioceptive information was available. The participants were required to navigate through the VE by either pedaling a bike or operating a computer mouse. The computer mouse allowed the participants to retain a sense of control over their movement trajectory but re- mained an unnatural form of navigation and provided minimal proprioceptive information.

\section{Method}

\section{Participants}

Forty undergraduate students from McMaster University participated in this study and were compensated with either course credit or money. All had normal or corrected-to-normal visual acuity.

\section{Materials}

The equipment and the paths were the same as those used in the VE condition of Experiments 2 and 3. However, one of the two conditions (the mouse condition) required the participants to navigate by operating a computer mouse instead of riding a bike.

\section{Procedure}

All the participants completed two conditions: (1) the bike condition and (2) the mouse condition. The learning phase and the testing phase involved the same VR interface as that in Experiment 2. The bike condition was essentially the same as the active condition in Experiment 3 and the VE condition in Experiment 2. The mouse condition also followed the same procedure as the bike condition; however, the participants navigated through the environment by operating a computer mouse instead of pedaling a bike. The participants moved forward through the environment by pressing and holding down the middle mouse button. They turned right by scrolling the mouse to the right and turned left by scrolling the mouse to the left. The participants stopped turning when they centered the mouse in the middle of the computer display. For both conditions, the participants were tested immediately following the completion of the learning phase, and testing was conducted in the same manner as in Experiment 3, with the exception that in the mouse condition, pointing was done via the mouse. The method used to measure the participants' pointing response estimations was the same as that used in the VE condition of Experiment 2. Pointing direction and reaction time were recorded.

\section{Results}

There was no significant difference between the performance of the participants who completed the bike conditionfirst and the performance of those who completed the mouse condition first [order effect: $F(1,38)=0.226, p>$ $.1]$, and therefore, the data were collapsed across learning order to simplify the results.

The raw score distributions of absolute pointing errors for the bike and the mouse conditions are shown in Figures 9A and 9B, respectively. Similarly, the raw score distributions of reaction times for the bike and the mouse conditions are shown in Figures 9C and 9D, respectively. All the distributions were unimodal. With regard to both errors and reaction times, in both the bike and the mouse conditions, the distributions were indistinguishable between aligned and contra-aligned orientations. This served to verify that there was minimal difference across the entire range of pointing errors across all the participants.

Both pointing errors and reaction times were subject to a $\log$ transformation to normalize the distribution. A $2 \times$ 2 repeated measures ANOVA ( 2 learning conditions [bike and mouse] $\times 2$ orientations [aligned and contra-aligned]) was conducted on the mean log pointing error and the mean $\log$ reaction time. Figures $10 \mathrm{~A}$ and $10 \mathrm{~B}$ illustrate mean $\log$ 
A

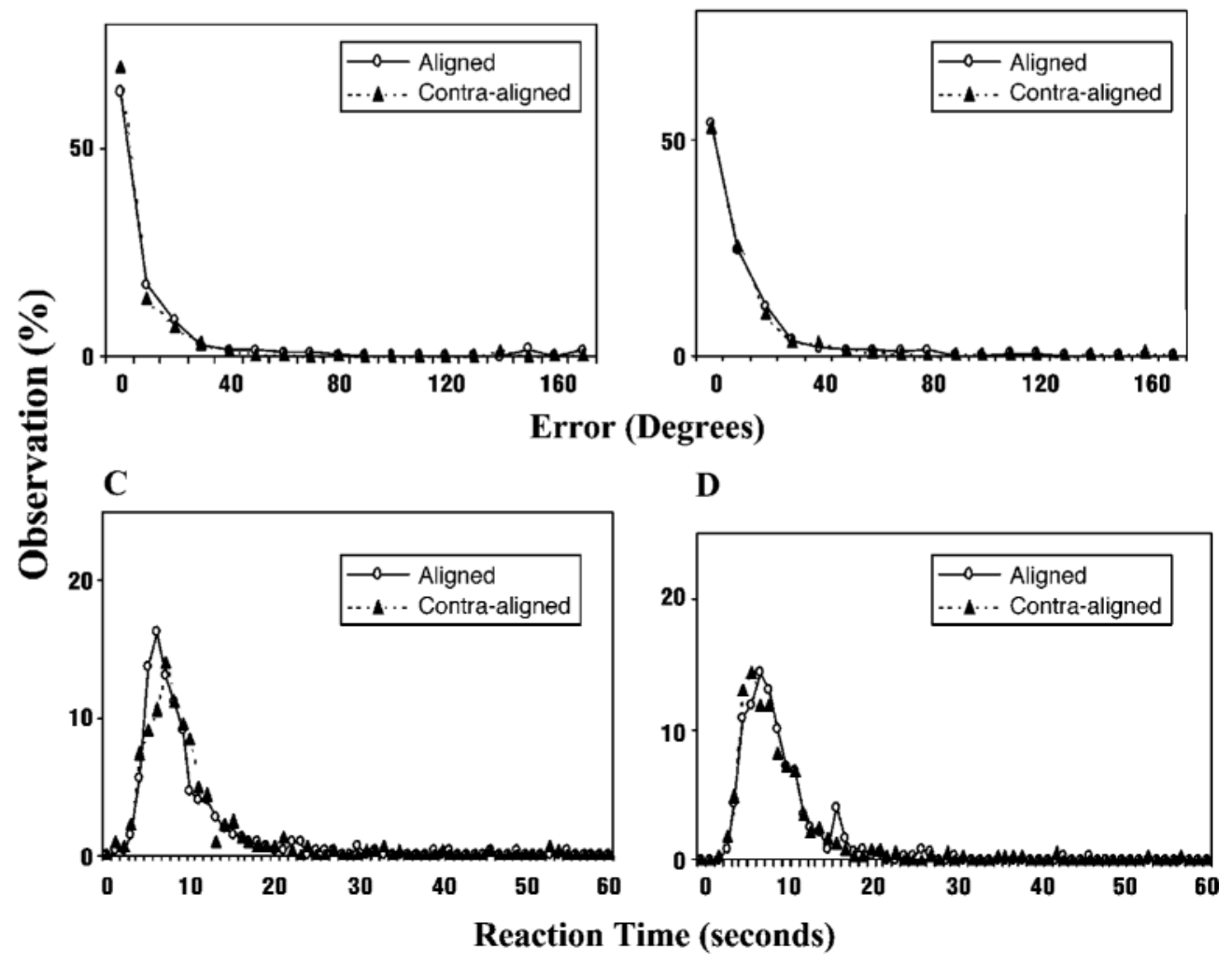

Figure 9. Experiment 4: pointing error distributions for (A) the bike condition and (B) the mouse condition and reaction time distributions for $(C)$ the bike condition and (D) the mouse condition.

pointing error and mean log reaction time collapsed across participants in each learning condition and each testing orientation.

\section{Pointing Error}

With regard to pointing error, a main effect of learning condition was observed $\left[F(1,39)=8.54, M S_{\mathrm{e}}=0.07, p<\right.$ $\left..05 ; \mathrm{M}_{\text {Bike }}=0.75, \mathrm{M}_{\text {Mouse }}=0.87\right]$. A main effect of orientation was not observed $\left[F(1,39)=0.42, M S_{\mathrm{e}}=0.05, p>\right.$ $\left..05 ; M_{\text {aligned }}=0.82, M_{\text {contra-aligned }}=0.80\right]$. Furthermore, an interaction effect between learning condition and orientation was not observed $\left[F(1,39)=2.24, M S_{\mathrm{e}}=0.04, p>.05\right]$. Therefore, no alignment effect was observed in either the bike or the mouse condition.

\section{Reaction Time}

With regard to reaction time, a main effect of learning condition was not observed $\left[F(1,39)=0.45, M S_{\mathrm{e}}=0.01\right.$, $\left.p>.05 ; \mathrm{M}_{\text {Bike }}=0.88, \mathrm{M}_{\text {Mouse }}=0.89\right]$. A main effect of orientation was not observed $\left[F(1,39)=0.15, M S_{\mathrm{e}}=0.01\right.$, $\left.p>.05 ; M_{\text {aligned }}=0.88, M_{\text {contra-aligned }}=0.89\right]$. Furthermore, an interaction effect was not observed between learning condition and orientation $\left[F(1,39)=1.21, M S_{\mathrm{e}}=0.01, p>\right.$ $.05]$. Therefore, no alignment effect was observed in either the bike or the mouse condition.

\section{Discussion}

Since no alignment effect was observed in either the mouse condition or the bike condition, it would seem that active navigation, regardless of the level of proprioceptive information provided, is sufficient for the development of an orientation-free representation.

The results from our mouse condition can be compared with results from other VR studies that have employed simple input devices, such as keyboards. For instance, Tlauka and Wilson (1996) investigated whether an alignment effect would be observed after participantshad learned and were tested in a VE via keyboard navigation. They then compared performance in this task with performance in another task in which learning occurred by examining a 2-D map. It was concluded that the reaction time of the directional judgments following training and testing in the VE did not demonstrate an alignment effect, whereas an alignment effect was demonstrated in the map condition. Although the conclusions they drew from their results were in agreement with what we found in our mouse condition, one needs to interpret such consistent findings with caution. The unique experimental design employed in their study makes it difficult to compare their findings with those from most other studies in which the alignment effect was examined, because their designation of the test orientation 

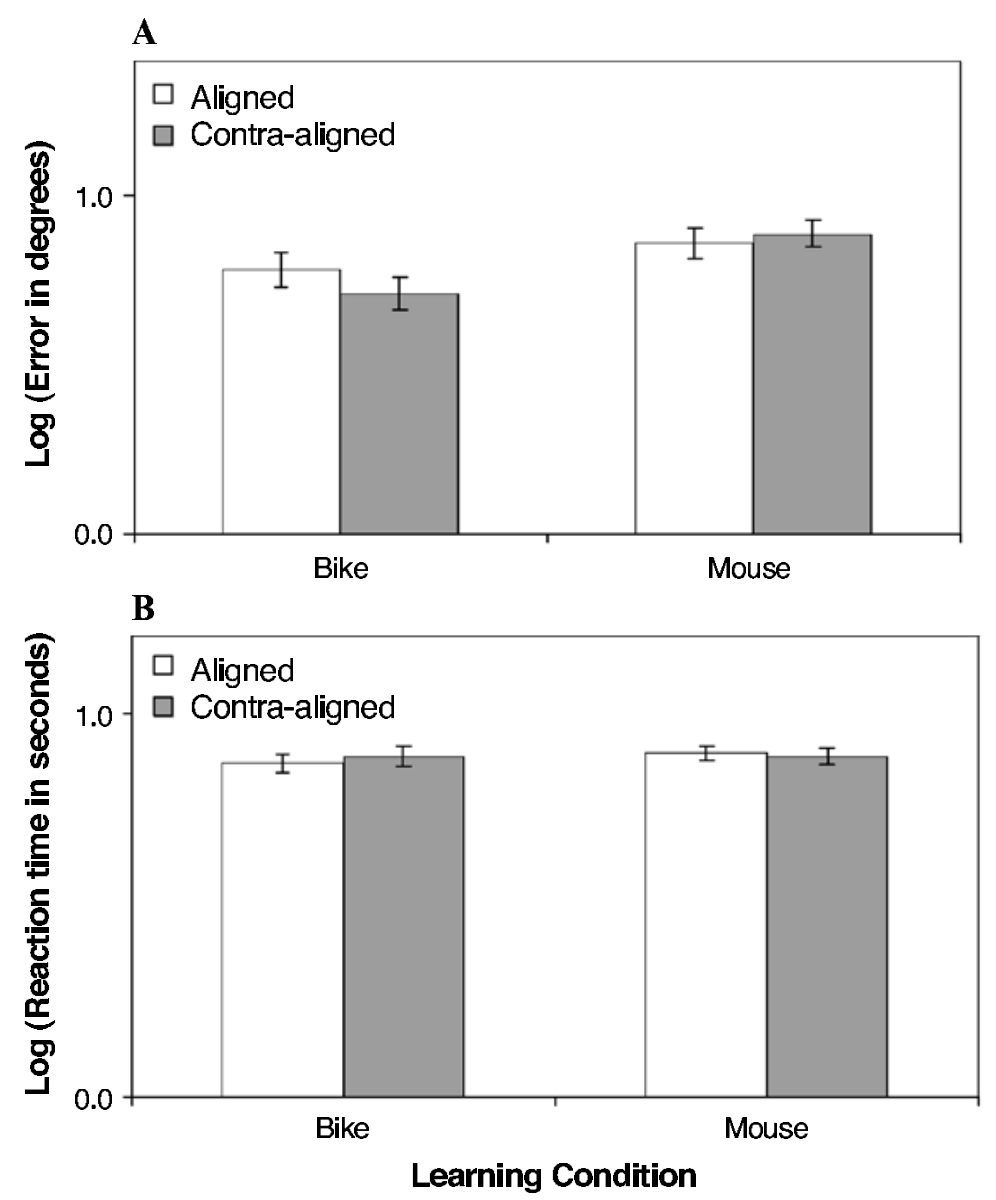

Figure 10. Experiment 4: (A) mean log pointing errors for the bike and mouse conditions and (B) mean log reaction time for the bike and mouse conditions. Error bars indicate standard errors.

as being aligned or contra-aligned was different from what is commonly used in the literature.

There may be a number of factors that may explain why an orientation-free representation was developed following active navigation in our study. During sighted navigation, what is experienced visually is tightly linked to the cues associated with self-motion, and thus the encoding of the environment may be enhanced as a result of this visual-sensorimotor interaction. For example, one's selfgenerated movements could enable one to anticipate the changing visual information that typically results from movement. During navigation, there are events that are particularly important for developing spatial knowledge of a specific environment-for instance, when changing directions. By actively controlling one's own movements, one may allocate more attentional resources to these critical events. Even the general sense of control during active movement could potentially enhance the learning experience.

It is conceivable that the predicted enhanced spatial knowledge that results from information gained from the simultaneous visual-sensorimotor interaction, as described above, does not require motor behavior to be executed in a manner that is identical to that in real-world navigation - that is, walking. Any motor behavior would serve the same purpose as long as it enables participants to interact with the environment. Therefore, we would predict that even a degraded form of motor behavior would result in a similar type of spatial representation. The results of Experiment 4 confirmed this supposition, so that an orientationfree representation was developed in both the mouse and the bike conditions.

It is important to note that although orientation-free representations were observed for both the bike condition and the mouse condition, the overall spatial performance in the bike condition was significantly more accurate than that in the mouse condition. In other words, proprioceptive information appeared to enable more precise spatial processing. This is consistent with a number of studies in which spatial performances have been compared using different types of VR interfaces (Arthur \& Hancock, 2001; Lathrop \& Kaiser, 2002; Waller, Hunt, \& Knapp, 1998). In these 
studies, researchers assessed how accurately and consistently participants could point to or move toward previously learned targets. Participants were significantly more accurate and more consistent in the real-world condition than in other conditions that did not involve a natural form of movement. In addition, Chance et al. (1998) found that when the visual display was updated in response to full body movements, spatial knowledge acquisition was facilitated, in comparison with conditions in which this updating was not possible.

\section{GENERAL DISCUSSION}

These experiments suggest that in both RE and VE active navigation conditions, participants consistently developed orientation-free representations, which is in contrast to the orientation-specific representations observed for both the map condition and the VE passive condition.

\section{Validating the Evidence Supporting Orientation-Free Representations}

The conclusions drawn with respect to any orientationfree representations observed were drawn from the lack of statistically significant differences observed between performances in aligned conditions and contra-aligned conditions. As is the case whenever one attempts to generate assumptions on the basis of null findings, it is important to ensure that the generalizations based on such results reflect a true phenomenon and not simply a statistical artifact. In order to verify the validity of nonsignificantresults, one must ensure both that an effective control is available for comparison and that a sufficient number of observations are included.

In the present discussion, there are a number of different factors indicating that the nonsignificant main effects reported here were not simply failures to reach significance. In Experiment 1, the null result reported for the VE condition is meaningful when this condition is compared with the $\mathrm{RE}$ and map conditions consisting of the same number of comparisons. The orientation-free and orientation-specific representations observed in the RE and map conditions reflect the typically observed and generally accepted dissociation. With regard to Experiment 2, it is justifiable to compare the null results reported for both conditions (RE and VE) with the null results observed for similar conditions in Experiment 1 since a within-subjects design was used in Experiment 2 (as opposed to the between-subjects design used in Experiment 1) and an increased number of observations were included in Experiment 2, thus making it a more sensitive measure.

Experiment 3 was particularly informative, since the results demonstrate that it is indeed possible for an alignment effect to be observed following passive learning in a VE. Not only does this serve to verify the statistical power observed for the null results in the active condition of Experiment 3, but also it serves as a comparison for the VE condition in Experiment 2 and the bike condition in Experiment 4 , which were all essentially equivalent. Thus, the null results obtained in all of these experiments can be interpreted as indicating the absence of an alignmenteffect, and not simply as reflecting a failure to reveal the effect.

For this same reason, if concern remains about the possibility that the lack of statistical significance observed in each of these conditions is due to an insufficient number of participants in each condition, the results of each of these equivalent conditions (VE in Experiment 2, active in Experiment 3, and bike in Experiment 4) could be combined, forming a total of 80 participants. Furthermore, with regard to Experiment 4, a substantial number of participants were included in the mouse condition, thus providing ample opportunity for the null hypothesis to be rejected. The fact that it failed to be rejected speaks to the strength of the result.

The general trend of failing to observe an alignment effect in the equivalent VE conditions (VE in Experiment 2, active in Experiment 3, and bike in Experiment 4) is not without exception. In Experiment 2, with regard to the reaction time results, the main effect of orientation showed a trend that approached statistical significance $(p=.07)$. It is conceivable that with additional observations, a significant difference between performances for aligned and contra-aligned orientations could be observed.

Even if such reaction time results were to turn out to be statistically significant, one must interpret these results in the context of the following related issues. First, as was indicated above, the measurement sensitivity for Experiment 2 should be stronger than that of Experiment 1, which demonstrated the lack of an alignment effect typically observed for the RE and VE conditions and a strong alignment effect for the map condition. The pointing error results observed for both the RE and the VE conditions in Experiment 2 are consistent with what was found in Experiment 1 . Second, the fact that no interaction effect was observed with regard to reaction time in Experiment 2 provides evidence that VE learning, like RE learning, results in orientation-free representations. Third, because the small effect of orientation observed in Experiment 2 applies only to reaction time, it is possible that the differences in reaction time in Experiment 2 may represent a reaction time/ accuracy tradeoff.

\section{Empirical Test for the Two Theories for Spatial Representation}

In our study, we empirically tested two theories that attempt to specify the particular factors that lead to the development of orientation-free representations versus orientation-specific representations. Our series of experiments addressed a number of issues that had not been thoroughly examined previously. Issues of particular interest to this discussion include the degree to which the number of vantage points experienced during navigation affects spatial representations, the effect of actively versus passively experiencing a path on the resulting spatial knowledge, and how different levels of proprioceptive information differentially affect the development of spatial representations. 


\section{The Multiple Vantage Points Theory}

As was previously mentioned, the differences observed following map learning, as compared with learning via navigation, have been attributed to the number of vantage points participants are exposed to during learning (Evans $\&$ Pezdek, 1980). The notion of vantage points could potentially include nonvisual components (i.e., body and head orientation), as well as visual components. With regard to the nonvisual components, by comparing the results of Experiments 1 and 2, we were able to determine how performance in situations in which participants were allowed only a single body orientation differed from that in those conditions that allowed participants to experience multiple body orientations. Inasmuch as orientation-free representations were observed in both of these experiments, these results provided compelling evidence that experiencing multiple body orientations during navigation is not required for developing orientation-free representations.

It is important to note that in the RE condition of Experiment 2 , the participants were still able to rotate their heads to view landmarks outside their direct field of view. Therefore, it remains possible that even though the participants were required to maintain a single body orientation, the fact that they were still able to turn their heads enabled the development of an orientation-free representation. Direct evidence regarding the effect of head orientation could be provided by comparing the results of Experiment 2 with a real-world navigation task in which both body and head orientation are held constant. Although this was not tested explicitly in the present study, the orientation-free representations observed following VE navigation, in which the participants did not experience multiple head orientations, suggest that multiple head orientations may not be necessary.

Vantage points can be experienced through nonvisual means, and they can also be experienced visually. One can, therefore, question the effect that multiple visual vantage points would have on the resulting spatial representation. We can address this issue by examining the results of the passive condition in Experiment 3, in which the participants experienced only visual information. If the multipleviewpoint theory holds true, we would expect that in the passive condition, an orientation-free representation would develop. However, in our study, an orientation-specific representation developed despite the fact that multiple vantage points were experienced-in this case, visually.

Collectively, this series of experiments clearly challenges the multiple vantage points theory. If the possibility that orientation-free representations are developed simply as a result of the greater number of vantage points is ruled out, the next logical explanation relates to the intrinsic properties of active navigation.

\section{Active Navigation and Its Possible Constituent Components}

When theories that account for the causal factors leading to the development of an orientation-free/specific representation are evaluated, our results strengthen the underly- ing assumptions of the primary learning theory. The main premise of this theory relies on the notion that different spatial representations are developed depending on the degree to which one interacts with their environment during learning.

The present study was the first to compare active learning to passive learning in the context of orientation specificity. It was demonstrated that the participants developed an orientation-specific representation when learning their environment passively, whereas an orientation-free representation was developed following active learning. Consequently, it would seem that there is something unique about the active nature of navigation that leads to the development of orientation-free representations.

After having determined that active navigation results in spatial representations that differ from passive learning, in Experiment 4 we sought to gain insight into the exact constituent components that make up active navigation. To do this, we first explored whether the degree of kinesthetic or proprioceptiveinput that was experienced affected the resulting spatial representations. The orientation-free representations that were developed in both the bike and the mouse conditions demonstrated that only a minimal level of proprioceptive information is necessary to build an orientation-free representation, provided that participants actively control their own movements. This result also demonstrates that the type of proprioceptive information that is provided is irrelevant, whether it is naturally linked to locomotion (i.e., legs) or not (i.e., hands). That said, one might argue that the bike setup of our VR interface does not provide completely natural proprioceptive information. However, the fact that the same type of representation was observed following RE and VE navigation in Experiment 2 makes this speculation insignificant. Therefore, it seems that overall, regardless of the amount or quality of proprioceptive information that was provided, the same type of spatial representation was developed.

\section{Orientation Specificity and Its Implications for Understanding Spatial Representations}

It is important to examine the degree to which one's spatial representation is orientation specific, as identified by whether or not spatial memory is dependent on the original orientation in which the spatial layout was learned. This property of spatial representations is one of the very few concrete properties that one can measure empirically and, thus, provides one of the major clues regarding how spatial representations are developed. Identifying the factors that cause observers to form orientation-free or orientationspecific representations of their external world is important in understanding the mechanisms that underlie spatial processing.

In terms of the functional properties of orientation-free and orientation-specific representations, each of these types of spatial representations may potentially serve a different purpose. When one forms an orientation-specific representation, it may be that spatial information is encoded 
as discrete picture-like events that are organized in terms of an egocentric, or viewer-centered, frame of reference. Such spatial knowledge can be retrieved with high accuracy only if it is recalled in the same orientation as that in which it is initially learned.

In contrast, when orientation-free representations are developed, observers are able to integrate spatial information over space and time and consequently form a unified knowledge of space independently of their body position and orientation (Presson et al., 1989). The spatial representations revealed in this case would appear to be based on an exocentric, or environment-centered, frame of reference. Indeed, an orientation-free representation would offer an advantage to the observer. Such representations are flexible enough that they provide one with the capability to more easily recognize scenes learned from a number of different perspectives and allows for the continuous updating of spatial knowledge during further environmental exploration. More important, flexible knowledge about an overall spatial layout would be useful in guiding locomotion in situations in which a novel path is chosen to reach a destination.

\section{Conclusions}

Orientation specificity is an important concept in the discussion of spatial processing. Orientation-free versus orientation-specific representation is one of the few functional distinctions between different ways humans represent spatial information. VR technology provides a unique testing tool to complement traditionally employed real-world navigation tasks and map-learning paradigms in evaluating human spatial representations. Such paradigms assist in one's understanding the nature of the mechanisms underlying the development of particular spatial representations. The key findings in this study indicate that orientation-free representations are developed to a large extent as a consequence of the interaction of information from different modalities and that this interaction occurs most effectively during active navigation.

\section{REFERENCES}

ARTHUR, E. J., \& HANCOCK, P. A. (2001). Navigation training in virtual environments. International Journal of Cognitive Ergonomics, 5, 387-400.

Bakker, N. H., Werkhoven, P. J., \& PAssenier, P. O. (1999). The effects of proprioceptive and visual feedback on geographical orientation in virtual environments. Presence: Teleoperators \& Virtual Environments, 8, 36-53.

Bakker, N. H., Werkhoven, P. J., \& Passenier, P. O. (2001). Calibrating visual path integration in VEs. Presence: Teleoperators \& Virtual Environments, 10, 216-224.

Chance, S. S., Gaunet, F., Beall, A. C., \& Loomis, J. M. (1998). Locomotion mode affects the updating of landmarks encountered during travel: The contribution of vestibular and proprioceptive inputs to path integration. Presence: Teleoperators \& Virtual Environments, 7, 168 178.

EAston, R. D., \& Sholl, M. J. (1995). Object-array structure, frames of reference, and retrieval of spatial knowledge. Journal of Experimental Psychology: Learning, Memory, \& Cognition, 21, 483-500.

Eichenbaum, H., \& Cohen, N. J. (2001). From conditioning to conscious recollection: Memory systems of the brain. Oxford: Oxford University Press.
Evans, G. W., \& Pezde K, K. (1980). Cognitive mapping: Knowledge of real-world distance and location information. Journal of Experimental Psychology: Learning, Memory, \& Cognition, 6, 13-24.

FARrelL, M. J., \& RobertSon, I. H. (1998). Mental rotation and automatic updating of body-centered spatial relationships. Journal of Experimental Psychology: Learning, Memory, \& Cognition, 24, 227 233.

FARrell, M. J., \& Thomson, J. A. (1998). Automatic spatial updating during locomotion without vision. Quarterly Journal of Experimental Psychology, 51A, 637-654.

Golledge, R. G. (1999). Wayfinding behavior. Baltimore: Johns Hopkins University Press.

Kearns, M. J., Warren, W. H., Duchon, A. P., \& Tarr, M. J. (2002). Path integration from optic flow and body senses in a homing task. Perception, 31, 349-374.

Klatzky, R. L., Loomis, J. M., Beall, A. C., Chance, S. S., \& GOLLEDGE, R. G. (1998). Spatial updating of self-position and orientation during real, imagined, and virtual locomotion. Psychological Science, 9, 293-298.

LATHRop, W. B., \& KAISER, M. K. (2002). Perceived orientation in physical and virtual environments: Changes in perceived orientation as a function of idiothetic information available. Presence: Teleoperators \& Virtual Environments, 11, 19-32.

Le vine, M., Jankovic, I. N., \& PAliJ, M. (1982). Principles of spatial problem solving. Journal of Experimental Psychology: General, 111, 157-175.

LoOmis, J. M., Blascovich, J. J., \& BEALl, A. C. (1999). Immersive virtual environment technology as a basic research tool in psychology. Behavior Research Methods, Instruments, \& Computers, 31, 557-564.

Loomis, J. M., Klatzky, R. L., Golledge, R. G., \& Philbeck, J. W. (1999). Human navigation by path integration. In R. G. Golledge (Ed.), Wayfinding behavior (pp. 125-151). Baltimore: Johns Hopkins University Press.

May, M., Peruch, P., \& SAVoyant, A. (1995). Navigating in a virtual environment with map-acquired knowledge: Encoding and alignment effects. Ecological Psychology, 7, 21-36.

Paliu, M., LeVine, M., \& KAHAN, T. (1984). The orientation of cognitive maps. Bulletin of the Psychonomic Society, 22, 105-108.

PERUCH, P., \& LAPIN, E. A. (1993). Route knowledge in different spatial frames of reference. Acta Psychologica, 84, 253-269.

Presson, C. C., Delange, N., \& HAZElrigg, M. D. (1987). Orientationspecificity in kinesthetic spatial learning: The role of multiple orientations. Memory \& Cognition, 15, 225-229.

Presson, C. C., Delange, N., \& Hazelrigg, M. D. (1989). Orientation specificity in spatial memory: What makes a path different from a map of the path? Journal of Experimental Psychology: Learning, Memory, \& Cognition, 15, 887-897.

Presson, C. C., \& HAzelrigG, M. D. (1984). Building spatial representations through primary and secondary learning. Journal of Experimental Psychology: Learning, Memory, \& Cognition, 10, 716-722.

Presson, C. C., \& Montello, D. R. (1994). Updating after rotational and translational body movements: Coordinate structure of perspective space. Perception, 23, 1447-1455.

Presson, C. C., \& Somerville, S. C. (1985). Beyond egocentrism: A new look at the beginnings of spatial representation. In H. M. Wellman (Ed.), Children's searching: The development of search skill and spatial representation (pp. 1-26). Hillsdale, NJ: Erlbaum.

Richardson, A. E., Montello, D. R., \& Hegarty, M. (1999). Spatial knowledge acquisition from maps and from navigation in real and virtual environments. Memory \& Cognition, 27, 741-750.

RIESER, J. J. (1989). Access to knowledge of spatial structure at novel points of observation. Journal of Experimental Psychology: Learning, Memory, \& Cognition, 15, 1157-1165.

Roskos-Ewoldsen, B., McNamara, T. P., Shelton, A. L., \& Carr, W. (1998). Mental representations of large and small spatial layouts are orientation dependent. Journal of Experimental Psychology: Learning, Memory, \& Cognition, 24, 215-226.

RosSANo, M. J., \& MOAK, J. (1998). Spatial representations acquired from computer models: Cognitive load, orientation-specificity and the acquisition of survey knowledge. British Journal of Psychology, 89, 481-497. 
Rossano, M. J., \& Warren, D. H. (1989). Misaligned maps lead to predictable errors. Perception, 18, 215-229.

Rossano, M. J., West, S. O., Robertson, T. J., Wayne, M. C., \& CHASE, R. B. (1999). The acquisition of route and survey knowledge from computer models. Journal of Environmental Psychology, 19, 101-115.

SHOLL, M. J. (1987). Cognitive maps as orienting schemata. Journal of Experimental Psychology: Learning, Memory, \& Cognition, 13, $615-$ 628.

Sholl, M. J., \& BARTEls, G. P. (2002). The role of self-to-object updating in orientation-free performance on spatial-memory tasks. Journal of Experimental Psychology: Learning, Memory, \& Cognition, 28, 422-436.

Sholl, M. J., \& Nolin, T. L. (1997). Orientation specificity in representations of place. Journal of Experimental Psychology: Learning, Memory, \& Cognition, 23, 1494-1507.

SIEGEL, A. W. (1981). The externalization of cognitive maps by children and adults: In search of ways to ask better questions. In L. S. Liben, A. Patterson, \& N. Newcombe (Eds.), Spatial representation and behavior across the life span: Theory and application (pp. 167-194). New York: Academic Press.

SimONS, D. J., \& WANG, R. F. (1998). Perceiving real-world viewpoint changes. Psychological Science, 9, 315-320.

Sun, H.-J., CAMPos, J. L., \& Chan, G. S. W. (2004). Multisensory integration in the estimation of relative path length. Experimental Brain Research, 154, 246-254.

Sun, H.-J., Campos, J. L., Chan, G. S. W. Young, M., \& Ellard, C.
(2004). The contributions of static visual cues, nonvisual cues, and optic flow in distance estimation. Perception, 33, 49-65.

Thorndyke, P. W., \& Hayes-Roth, B. (1982). Differences in spatial knowledge acquired from maps and navigation. Cognitive Psychology, 14, 560-589.

Tlauka, M., \& Wilson, P. N. (1996). Orientation-free representations from navigation through a computer-simulated environment. Environment \& Behavior, 28, 647-664.

WAller, D., Hunt, E., \& KNAPP, D. (1998). The transfer of spatial knowledge in virtual environment training. Presence: Teleoperators \& Virtual Environments, 7, 129-143.

Warren, D. H., Rossano, M. J., \& Wear, T. D. (1990). Perception of map environment correspondence: The roles of features and alignment. Ecological Psychology, 2, 131-150.

WiLSON, P. N. (1997). Use of virtual reality computing in spatial learning research. In N. Foreman \& R. Gillett (Eds.), A handbook of spatial research paradigms and methodologies: Vol. 1. Spatial cognition in the child and adult (pp. 181-206). Hove, U.K.: Psychology Press.

WILSON, P. N. (1999). Active exploration of a virtual environment does not promote orientation or memory for objects. Environment \& Behavior, 31, 752-763.

Wilson, P. N., Foreman, N., Gillett, R., \& Stanton, D. (1997). Active versus passive processing of spatial information in a computersimulated environment. Ecological Psychology, 9, 207-222.

(Manuscript received December 19, 2002; revision accepted for publication August 14, 2003.) 